\title{
THE WAVE EQUATION ON DAMEK-RICCI SPACES
}

\author{
JEAN-PHILIPPE ANKER, VITTORIA PIERFELICE, AND MARIA VALLARINO
}

ABSTRACT. We study the dispersive properties of the wave equation associated with the shifted Laplace-Beltrami operator on Damek-Ricci spaces, and deduce Strichartz estimates for a large family of admissible pairs. As an application, we obtain global well-posedness results for the nonlinear wave equation.

\section{INTRODUCTION}

The aim of this paper is to study the dispersive properties of the linear wave equation on Damek-Ricci spaces and their application to nonlinear Cauchy problems.

For the linear wave equation on $\mathbb{R}^{n}$

$$
\left\{\begin{array}{l}
\partial_{t}^{2} u(t, x)-\Delta_{x} u(t, x)=F(t, x), \\
u(0, x)=f(x), \\
\left.\partial_{t}\right|_{t=0} u(t, x)=g(x),
\end{array}\right.
$$

the theory is well established; the dispersive $L^{1} \rightarrow L^{\infty}$ estimates are classical, while Strichartz estimates were proved by [16] and [23]. These estimates serve as main tools to study the corresponding nonlinear problems and to prove local and global existence with either small or large initial data. In particular, for the semilinear wave equation

$$
\left\{\begin{array}{l}
\partial_{t}^{2} u(t, x)-\Delta_{x} u(t, x)=F(u t(, x)), \\
u(0, x)=f(x) \\
\left.\partial_{t}\right|_{t=0} u(t, x)=g(x)
\end{array}\right.
$$

with

$$
F(u) \sim|u|^{\gamma} \text { near } 0,
$$

a fairly complete theory of well-posedness for small initial data exists. The results depend on the space dimension $n$. After the pioneering work [21] of John in dimension $n=3$, Strauss conjectured in 30 , that the problem (2) is globally well-posed for small initial data provided

$$
\gamma>\gamma_{0}(n)=\frac{1}{2}+\frac{1}{n-1}+\sqrt{\left(\frac{1}{2}+\frac{1}{n-1}\right)^{2}+\frac{2}{n-1}} \quad(n \geq 2) .
$$

Date: October 30, 2018.

2000 Mathematics Subject Classification. 35L05, 43A85, 58J45; 22E30, 35L71, 43A90, 47J35, 58D25.

Key words and phrases. Damek-Ricci spaces, semilinear wave equation, dispersive estimate, Strichartz estimate, global well-posedness.

This work was mostly carried out while the third author was a CNRS postdoc at the Fédération Denis Poisson Orléans-Tours. 
The negative part of the conjecture was verified in [29] by Sideris, who proved blow up for generic data when $\gamma<\gamma_{0}(n)$ (and nonlinearities satisfying $F(u) \gtrsim|u|^{\gamma}$ ). The positive part of the conjecture was also verified for any dimension in several steps (see e.g. [24] [26],[15], [10], as well as [14] for a survey and [11], [12] for related results).

Several attemps have been made to extend Strichartz estimates for dispersive equations from Euclidean spaces to other settings. In this paper we consider the shifted wave equation

$$
\left\{\begin{array}{l}
\partial_{t}^{2} u(t, x)-\left(\Delta_{S}+Q^{2} / 4\right) u(t, x)=F(t, x) \\
u(0, x)=f(x) \\
\left.\partial_{t}\right|_{t=0} u(t, x)=g(x),
\end{array}\right.
$$

on Damek-Ricci spaces $S$ (also known as harmonic NA groups). Recall that these spaces are solvable extensions $S=N \ltimes \mathbb{R}^{+}$of Heisenberg type groups $N$, equipped with an invariant Riemannian structure; $\Delta_{S}$ denotes the associated Laplace-Beltrami operator, whose $L^{2}$ spectrum is the half line $\left(-\infty ;-Q^{2} / 4\right]$, and $Q$ the homogeneous dimension of $N$. As Riemannian manifolds, these solvable Lie groups include all symmetric spaces of the noncompact type and rank one; they are all harmonic but most of them are not symmetric, thus providing counterexamples to the Lichnerowicz conjecture [8]. We refer to Section 2 for more details about their structure and analysis thereon.

The Cauchy problem (5) was considered by Tataru [31] and by Ionescu [20. Tataru obtained sharp dispersive $L^{q^{\prime}} \rightarrow L^{q}$ estimates for the operators

$$
\cos \left(t \sqrt{-\Delta_{S}-Q^{2} / 4}\right) \text { and } \frac{\sin \left(t \sqrt{-\Delta_{S}-Q^{2} / 4}\right)}{\sqrt{-\Delta_{S}-Q^{2} / 4}}
$$

when $S$ is a real hyperbolic space, while Ionescu investigated $L^{q} \rightarrow L^{q}$ estimates for these operators when $S$ is a rank one symmetric space.

In [4] we derived Strichartz estimates for the Cauchy problem (15) when $S$ is a real hyperbolic space. Our aim here is to extend the results obtained in [4] to the larger class of Damek-Ricci spaces. The difficulty is due to the fact that Damek-Ricci spaces are nonsymmetric in general, so that some of the proofs given in [4] do not work in this context. Despite this difficulty, we are able to obtain Strichartz estimates for solutions to the Cauchy problem (5). Corresponding results for the the Schrödinger equation were obtained [2] and [3] (see also [27]).

In Section 7 we apply our Strichartz estimates to obtain global well-posedness results for the nonlinear wave equation with small initial data and low regularity. Notice that this result is new even for hyperbolic spaces, since in [4] we only discussed local wellposedness. An interesting new feature, which differentiates our results from the Euclidean case, is the absence of a lower critical exponent for power-like nonlinearities on DamekRicci spaces. Indeed, for $\gamma>1$ arbitrarily close to 1 , we are able to prove global existence for the problem

$$
\left\{\begin{array}{l}
\partial_{t}^{2} u(t, x)-\left(\Delta_{S}+Q^{2} / 4\right) u(t, x)=F(u(t, x)), \\
u(0, x)=f(x), \\
\left.\partial_{t}\right|_{t=0} u(t, x)=g(x),
\end{array}\right.
$$


with small initial data and nonlinearities $F$ satisfying

$$
|F(u)| \leq C|u|^{\gamma} \text { and }|F(u)-F(v)| \leq C\left(|u|^{\gamma-1}+|v|^{\gamma-1}\right)|u-v| .
$$

Recall that Tataru 31] proved global existence on hyperbolic spaces for small smooth initial data, provided the power $\gamma$ is greater than the Strauss critical exponent (44). Thus, by combining our results with [31, we see that the Cauchy problem (66) is well posed for small smooth initial data and any power $\gamma>1$. Notice moreover that in Theorem (17.2) we allow for small initial data with low regularity, arbitrarily close to the critical one in the Euclidean case, which is determined by concentration and scaling arguments.

\section{DAMEK-RICCI SPACES}

In this section we recall the definition of $H$-type groups, describe their Damek-Ricci extensions, and recall the main results of spherical analysis on these spaces. For the details we refer the reader to [1, 6, 17, 8, 9, 28].

Let $\mathfrak{n}$ be a Lie algebra equipped with an inner product $\langle\cdot, \cdot\rangle$ and denote by $|\cdot|$ the corresponding norm. Let $\mathfrak{v}$ and $\mathfrak{z}$ be complementary orthogonal subspaces of $\mathfrak{n}$ such that $[\mathfrak{n}, \mathfrak{z}]=\{0\}$ and $[\mathfrak{n}, \mathfrak{n}] \subseteq \mathfrak{z}$. According to Kaplan [22], the algebra $\mathfrak{n}$ is of $H$-type if, for every $Z$ in $\mathfrak{z}$ of unit length, the map $J_{Z}: \mathfrak{v} \rightarrow \mathfrak{v}$, defined by

$$
\left\langle J_{Z} X, Y\right\rangle=\langle Z,[X, Y]\rangle \quad \forall X, Y \in \mathfrak{v},
$$

is orthogonal. The connected and simply connected Lie group $N$ associated to $\mathfrak{n}$ is called an $H$-type group. We identify $N$ with its Lie algebra $\mathfrak{n}$ via the exponential map

$$
\begin{aligned}
\mathfrak{v} \times \mathfrak{z} & \longrightarrow N \\
(X, Z) & \longmapsto \exp (X+Z) .
\end{aligned}
$$

Thus multiplication in $N$ reads

$$
(X, Z)\left(X^{\prime}, Z^{\prime}\right)=\left(X+X^{\prime}, Z+Z^{\prime}+\frac{1}{2}\left[X, X^{\prime}\right]\right) \quad \forall X, X^{\prime} \in \mathfrak{v} \quad \forall Z, Z^{\prime} \in \mathfrak{z} .
$$

The group $N$ is a two-step nilpotent group with Haar measure $d X d Z$. The number $Q=\frac{m}{2}+k$, where $m$ and $k$ denote the dimensions of $\mathfrak{v}$ and $\mathfrak{z}$ respectively, is called the homogeneous dimension of $N$.

Let $S$ be the semidirect product $S=N \ltimes \mathbb{R}^{+}$, defined by

$$
(X, Z, a)\left(X^{\prime}, Z^{\prime}, a^{\prime}\right)=\left(X+a^{\frac{1}{2}} X^{\prime}, Z+a Z^{\prime}+\frac{1}{2} a^{\frac{1}{2}}\left[X, X^{\prime}\right], a a^{\prime}\right)
$$

for all $(X, Z, a),\left(X^{\prime}, Z^{\prime}, a^{\prime}\right) \in S$. We shall denote by $n$ the dimension $m+k+1$ of $S$. Notice that $m$ is an even number $\geq 2$ and we shall always assume that $k \geq 1$ (the case when $k=0$ corresponds to real hyperbolic spaces and has been investigated in [4]). This implies that the dimension of the space $S$ is $n \geq 4$.

The group $S$ is nonunimodular. Indeed the right and left Haar measures on $S$ are given respectively by

$$
d \rho(X, Z, a)=a^{-1} d X d Z d a \quad \text { and } \quad d \mu(X, Z, a)=a^{-(Q+1)} d X d Z d a .
$$

Then the modular function is $\delta(X, Z, a)=a^{-Q}$. 
We equip $S$ with the left invariant Riemannian metric induced by the inner product

$$
\left\langle(X, Z, \ell),\left(X^{\prime}, Z^{\prime}, \ell^{\prime}\right)\right\rangle=\left\langle X, X^{\prime}\right\rangle+\left\langle Z, Z^{\prime}\right\rangle+\ell \ell^{\prime},
$$

on the Lie algebra $\mathfrak{s}$ of $S$. For every $x \in S$, we shall denote by $r(x)$ the distance between the point $x$ and the identity $e$ of $S$ and by $a(x)$ the $A$-component of $x$, i.e. the element $a(x) \in \mathbb{R}^{+}$such that $x=(X, Z, a(x))$, with $X \in \mathfrak{v}, Z \in \mathfrak{z}$. The following useful inequality holds (see [1, formula (1.20)]) :

$$
|\log a(x)| \leq r(x) \quad \forall x \in S .
$$

The Riemannian measure is the left Haar measure $\mu$ introduced above and we denote by $\Delta_{S}$ the Laplace-Beltrami operator associated with this Riemannian structure on $S$.

A radial function on $S$ is a function that depends only on the distance from the identity. If $f$ is radial, then by [1, formula (1.16)]

$$
\int_{S} d \mu f=\int_{0}^{\infty} d r f(r) V(r)
$$

where

$$
V(r)=2^{m+k} \sinh ^{m+k} \frac{r}{2} \cosh ^{k} \frac{r}{2} \quad \forall r \in \mathbb{R}^{+} .
$$

Let $\pi$ denote the radialisation operator defined in [5, page 150] which associates to each function $f$ in $C^{\infty}(S)$ a radial function on $S$. More precisely,

$$
\pi f(r)=\text { const. } \int_{\partial B(\mathfrak{s})} d \sigma f(r \sigma) \quad \forall r \in \mathbb{R}^{+},
$$

where $\partial B(\mathfrak{s})$ is the unit sphere in $\mathfrak{s}$ and $d \sigma$ denotes the surface measure on it.

The spherical functions $\varphi_{\lambda}$ on $S$ are normalized eigenfunctions of $\Delta_{S}$ :

$$
\left\{\begin{array}{l}
\Delta_{S} \varphi_{\lambda}=-\left(\lambda^{2}+\frac{Q^{2}}{4}\right) \varphi_{\lambda}, \\
\varphi_{\lambda}(e)=1
\end{array}\right.
$$

where $\lambda \in \mathbb{C}$ (see [1, formula (2.6)]). In the sequel we shall use various properties of the spherical functions, which we now summarize. We refer to [1, 9] for more details.

All spherical functions are of the form

$$
\varphi_{\lambda}=\pi\left(\delta^{i \lambda / Q-1 / 2}\right)=\pi\left(a(\cdot)^{-i \lambda+Q / 2}\right) \quad \forall \lambda \in \mathbb{C},
$$

where $\delta$ is the modular function. This easily implies that

$$
\left|\varphi_{\lambda}(r)\right| \lesssim \varphi_{0}(r) \quad \forall \lambda \in \mathbb{C}, \forall r \in \mathbb{R}^{+} .
$$

Moreover, it is well known that

$$
\varphi_{0}(r) \lesssim(1+r) e^{-\frac{Q}{2} r} \quad \forall r \in \mathbb{R}^{+} .
$$

The asymptotic behavior of the spherical functions is given by

$$
\varphi_{\lambda}(r)=\mathbf{c}(\lambda) \Phi_{\lambda}(r)+\mathbf{c}(-\lambda) \Phi_{-\lambda}(r) \quad \forall \lambda \in \mathbb{C} \backslash \frac{i}{2} \mathbb{Z},
$$


where

$$
\mathbf{c}(\lambda)=\Gamma\left(\frac{n}{2}\right) 2^{Q-2 i \lambda} \frac{\Gamma(2 i \lambda)}{\Gamma\left(i \lambda+\frac{Q}{2}\right) \Gamma\left(i \lambda+\frac{m}{4}+\frac{1}{2}\right)}
$$

and

$$
\Phi_{\lambda}(r)=\left(2 \cosh \frac{r}{2}\right)^{i 2 \lambda-Q}{ }_{2} F_{1}\left(\frac{Q}{2}-i \lambda, \frac{m}{4}-\frac{1}{2}-i \lambda ; 1-2 i \lambda ;\left(\cosh \frac{r}{2}\right)^{-2}\right)
$$

(see [25, pp. 7-8]). On one hand, $\Phi_{\lambda}$ is another radial eigenfunction of $\Delta_{S}$ for the same eigenvalue $-\left(\lambda^{2}+\frac{Q^{2}}{4}\right)$, i.e.

$$
\begin{aligned}
0 & =\left\{\Delta_{S}+\frac{Q^{2}}{4}+\lambda^{2}\right\} \Phi_{\lambda}(r)=\left\{\partial_{r}^{2}+\frac{V^{\prime}(r)}{V(r))} \partial_{r}+\frac{Q^{2}}{4}+\lambda^{2}\right\} \Phi_{\lambda}(r) \\
& =V(r)^{-\frac{1}{2}}\left\{\partial_{r}^{2}-\omega(r)+\lambda^{2}\right\}\left\{V(r)^{\frac{1}{2}} \Phi_{\lambda}(r)\right\},
\end{aligned}
$$

where

$$
\begin{aligned}
\omega(r) & =V(r)^{-\frac{1}{2}} \partial_{r}^{2} V(r)^{\frac{1}{2}}-\frac{Q^{2}}{4} \\
& =\frac{1}{4} \frac{m}{2}(Q-1)\left(\sinh \frac{r}{2}\right)^{-2}+\frac{k}{2}\left(\frac{k}{2}-1\right)(\sinh r)^{-2} \\
& =\sum_{j=1}^{+\infty} \omega_{j} e^{-j r} \quad \text { with } \omega_{j}=\mathrm{O}(j) .
\end{aligned}
$$

On the other hand, the function $\Phi_{\lambda}$ can be expanded as follows :

$$
\begin{aligned}
\Phi_{\lambda}(r) & =\sum_{\ell=0}^{+\infty} \frac{\Gamma(Q / 2-i \lambda+\ell)}{\Gamma(Q / 2-i \lambda)} \frac{\Gamma(m / 4+1 / 2-i \lambda+\ell)}{\Gamma(m / 4+1 / 2-i \lambda)} \frac{\Gamma(1-2 i \lambda+\ell)}{\Gamma(1-2 i \lambda)} \frac{2^{2 \ell}}{\ell !}\left(2 \cosh \frac{r}{2}\right)^{2 i \lambda-Q-2 \ell} \\
& =2^{-\frac{k}{2}} V(r)^{-\frac{1}{2}} \sum_{\ell=0}^{+\infty} \Gamma_{\ell}(\lambda) e^{(i \lambda-\ell) r} \quad \text { as } r \rightarrow+\infty .
\end{aligned}
$$

By combining (13), (14), (15), the coefficients $\Gamma_{\ell}$ are shown to satisfy the recurrence formula

$$
\left\{\begin{array}{l}
\Gamma_{0}=1, \\
\ell(\ell-i 2 \lambda) \Gamma_{\ell}(\lambda)=\sum_{j=0}^{\ell-1} \omega_{\ell-j} \Gamma_{j}(\lambda) \quad \forall \ell \in \mathbb{N}^{*} .
\end{array}\right.
$$

It is well known (see e.g. [5, Theorem 3.2]) that there exist nonnegative constants $C$ and $d$ such that

$$
\left|\Gamma_{\ell}(\lambda)\right| \leq C(1+\ell)^{d},
$$

for all $\ell \in \mathbb{N}$ and for all $\lambda \in \mathbb{C}$ with $\operatorname{Im} \lambda \geq-|\operatorname{Re} \lambda|$. We shall need the following improved estimates.

Lemma 2.1. Let $0<\varepsilon<1$ and $\Omega_{\varepsilon}=\left\{\lambda \in \mathbb{C}|| \operatorname{Re} \lambda|\leq \varepsilon| \lambda \mid, \operatorname{Im} \lambda \leq-\frac{1-\varepsilon}{2}\right\}$. Then, there exists a positive constant $d$ and, for every $h \in \mathbb{N}$, a positive constant $C$ such that

$$
\left|\partial_{\lambda}^{h} \Gamma_{\ell}(\lambda)\right| \leq C \ell^{d}(1+|\lambda|)^{-h-1} \quad \forall \ell \in \mathbb{N}^{*}, \forall \lambda \in \mathbb{C} \backslash \Omega_{\varepsilon} .
$$

Proof. The case $h \in \mathbb{N}^{*}$ follows by Cauchy's formula from the case $h=0$, that we prove now. On one hand, there exists $A \geq 0$ such that

$$
\left|\omega_{j}\right| \leq A j \quad \forall j \in \mathbb{N}^{*} .
$$


On the other hand, there exists $B>0$ such that

$$
|\ell-i 2 \lambda| \geq B \max \{\ell, 1+|\lambda|\} \quad \forall \ell \in \mathbb{N}^{*}, \forall \lambda \in \mathbb{C} \backslash \Omega_{\varepsilon} .
$$

Choose $C=2 A / B$ and $d \geq 1$ such that $d+1 \geq C$. For $\ell=1$, we have $\Gamma_{1}(\lambda)=\frac{\omega_{1}}{1-i 2 \lambda}$, which implies

$$
\left|\Gamma_{1}(\lambda)\right| \leq \frac{A}{B} \frac{1}{1+|\lambda|} \leq \frac{C}{1+|\lambda|}
$$

as required. For $\ell>1$, we have

which implies

$$
\Gamma_{\ell}(\lambda)=\frac{\omega_{\ell}}{\ell(\ell-i 2 \lambda)}+\frac{1}{\ell(\ell-i 2 \lambda)} \sum_{0<j<\ell} \omega_{\ell-j} \Gamma_{j}(\lambda)
$$

$$
\begin{aligned}
\left|\Gamma_{\ell}(\lambda)\right| & \leq \frac{A}{B} \frac{\ell}{1+|\lambda|}+\frac{A}{B} \frac{1}{\ell^{2}} \sum_{0<j<\ell}(\ell-j) \frac{C j^{d}}{1+|\lambda|} \\
& \leq \frac{C}{2} \frac{\ell^{d}}{1+|\lambda|}+\frac{C}{2} \frac{\ell^{d}}{1+|\lambda|} \frac{C}{\ell} \sum_{0<j<\ell}\left(\frac{j}{\ell}\right)^{d} \\
& \leq C \frac{\ell^{d}}{1+|\lambda|} .
\end{aligned}
$$

The spherical Fourier transform $\mathcal{H} f$ of an integrable radial function $f$ on $S$ is defined by

$$
\mathcal{H} f(\lambda)=\int_{S} d \mu f \varphi_{\lambda}
$$

For suitable radial functions $f$ on $S$, an inversion formula and a Plancherel formula hold:

$$
f(x)=c_{S} \int_{0}^{\infty} d \lambda|\mathbf{c}(\lambda)|^{-2} \mathcal{H} f(\lambda) \varphi_{\lambda}(x) \quad \forall x \in S,
$$

and

$$
\int_{S} d \mu|f|^{2}=c_{S} \int_{0}^{\infty} d \lambda|\mathbf{c}(\lambda)|^{-2}|\mathcal{H} f(\lambda)|^{2}
$$

where the constant $c_{S}$ depends only on $m$ and $k$. It is well known that

$$
|\mathbf{c}(\lambda)|^{-2} \lesssim|\lambda|^{-2}(1+|\lambda|)^{n-3} \quad \forall \lambda \in \mathbb{R} .
$$

In the sequel we shall use the fact that $\mathcal{H}=\mathcal{F} \circ \mathcal{A}$, where $\mathcal{A}$ denotes the Abel transform and $\mathcal{F}$ denotes the Fourier transform on the real line. Actually we shall use the factorization $\mathcal{H}^{-1}=\mathcal{A}^{-1} \circ \mathcal{F}^{-1}$. For later use, let us recall the inversion formulae for the Abel transform [1, formula (2.24)], which involve the differential operators

$$
\mathcal{D}_{1}=-\frac{1}{\sinh r} \frac{\partial}{\partial r} \quad \text { and } \quad \mathcal{D}_{2}=-\frac{1}{\sinh (r / 2)} \frac{\partial}{\partial r} .
$$

If $k$ is even, then

$$
\mathcal{A}^{-1} f(r)=a_{S}^{e} \mathcal{D}_{1}^{k / 2} \mathcal{D}_{2}^{m / 2} f(r)
$$

where $a_{S}^{e}=2^{-(3 m+k) / 2} \pi^{-(m+k) / 2}$, while, if $k$ is odd, then

$$
\mathcal{A}^{-1} f(r)=a_{S}^{o} \int_{r}^{\infty} \mathcal{D}_{1}^{(k+1) / 2} \mathcal{D}_{2}^{m / 2} f(s) \mathrm{d} \nu(s),
$$

where $a_{S}^{o}=2^{-(3 m+k) / 2} \pi^{-n / 2}$ and $\mathrm{d} \nu(s)=(\cosh s-\cosh r)^{-1 / 2} \sinh s \mathrm{~d} s$. 


\section{Sobolev SPACES AND CONSERVATION OF ENERGY}

Let us first introduce inhomogeneous Sobolev spaces on a Damek-Ricci space, which will be involved in the conservation laws, in the dispersive estimates and in the Strichartz estimates for the shifted wave equation. We refer to [32] for more details about function spaces on Riemannian manifolds.

Let $1<q<\infty$ and $\sigma \in \mathbb{R}$. By definition, $H_{q}^{\sigma}(S)$ is the image of $L^{q}(S)$ under $\left(-\Delta_{S}\right)^{-\frac{\sigma}{2}}$ (in the space of distributions on $S$ ), equipped with the norm

$$
\|f\|_{H_{q}^{\sigma}}=\left\|\left(-\Delta_{S}\right)^{\frac{\sigma}{2}} f\right\|_{L^{q}} .
$$

In this definition, we may replace $-\Delta_{S}$ by $-\Delta_{S}-\frac{Q^{2}}{4}+\frac{\widetilde{Q}^{2}}{4}$, where $\widetilde{Q}>Q$ and we set

$$
\widetilde{D}=\left(-\Delta_{S}-\frac{Q^{2}}{4}+\frac{\widetilde{Q}^{2}}{4}\right)^{\frac{1}{2}} .
$$

Thus $H_{q}^{\sigma}(S)=\widetilde{D}^{-\sigma} L^{q}(S)$ and $\|f\|_{H_{q}^{\sigma}} \sim\left\|\widetilde{D}^{\sigma} f\right\|_{L^{q}}$. If $\sigma=N$ is a nonnegative integer, then $H_{q}^{\sigma}(S)$ coincides with the Sobolev space

$$
W^{N, q}(S)=\left\{f \in L^{q}(S) \mid \nabla^{j} f \in L^{q}(S) \forall 1 \leq j \leq N\right\}
$$

defined in terms of covariant derivatives and equipped with the norm

$$
\|f\|_{W^{N, q}}=\sum_{j=0}^{N}\left\|\nabla^{j} f\right\|_{L^{q}} .
$$

By following the same proof of [4, Proposition 3.1] we obtain the following Sobolev embedding Theorem.

Proposition 3.1. Let $1<q_{1}<q_{2}<\infty$ and $\sigma_{1}, \sigma_{2} \in \mathbb{R}$ such that $\sigma_{1}-\frac{n}{q_{1}} \geq \sigma_{2}-\frac{n}{q_{2}}$. Then

$$
H_{q_{1}}^{\sigma_{1}}(S) \subset H_{q_{2}}^{\sigma_{2}}(S) \text {. }
$$

By this inclusion, we mean that there exists a constant $C>0$ such that

$$
\|f\|_{H_{q_{2}}^{\sigma_{2}}} \leq C\|f\|_{H_{q_{1}}^{\sigma_{1}}} \quad \forall f \in C_{c}^{\infty}(S) .
$$

Beside the $L^{q}$ Sobolev spaces $H_{q}^{\sigma}(S)$, our analysis of the shifted wave equation on $S$ involves the following $L^{2}$ Sobolev spaces:

$$
H^{\sigma, \tau}(S)=\widetilde{D}^{-\sigma} D^{-\tau} L^{2}(S),
$$

where $D=\left(-\Delta_{S}-\frac{Q^{2}}{4}\right)^{\frac{1}{2}}, \sigma \in \mathbb{R}$ and $\tau<\frac{3}{2}$ (actually we are only interested in the cases $\tau=0$ and $\left.\tau= \pm \frac{1}{2}\right)$. Notice that

$$
\begin{cases}H^{\sigma, \tau}(S)=H_{2}^{\sigma}(S) & \text { if } \tau=0, \\ H^{\sigma, \tau}(S) \subset H_{2}^{\sigma+\tau}(S) & \text { if } \tau<0, \\ H^{\sigma, \tau}(S) \supset H_{2}^{\sigma+\tau}(S) & \text { if } 0<\tau<\frac{3}{2} .\end{cases}
$$

Lemma 3.2. If $0<\tau<\frac{3}{2}$, then

$$
H^{\sigma, \tau}(S) \subset H_{2}^{\sigma+\tau}(S)+H_{2^{+}}^{\infty}(S),
$$

where $H_{2^{+}}^{\infty}(S)=\bigcap_{\substack{s \in \mathbb{R} \\ q>2}} H_{q}^{s}(S)$ (recall that $H_{q}^{s}(S)$ is decreasing as $q \searrow 2$ and $\left.s \nearrow+\infty\right)$. 
Proof. See [4, Lemma 3.2].

Let us next introduce the energy

$$
E(t)=\frac{1}{2} \int_{S} d \mu(x)\left\{\left|\partial_{t} u(t, x)\right|^{2}+\left|D_{x} u(t, x)\right|^{2}\right\}
$$

for solutions to the homogeneous Cauchy problem

$$
\left\{\begin{array}{l}
\partial_{t}^{2} u-\left(\Delta_{S}+\frac{Q^{2}}{4}\right) u=0 \\
u(0, x)=f(x) \\
\left.\partial_{t}\right|_{t=0} u(t, x)=g(x) .
\end{array}\right.
$$

It is easily verified that $\partial_{t} E(t)=0$, hence (22) is conserved. In other words, for every time $t$ in the interval of definition of $u$,

$$
\left\|\partial_{t} u(t, x)\right\|_{L_{x}^{2}}^{2}+\left\|D_{x} u(t, x)\right\|_{L_{x}^{2}}^{2}=\|g\|_{L^{2}}^{2}+\|D f\|_{L^{2}}^{2} .
$$

Let $\sigma \in \mathbb{R}$ and $\tau<\frac{3}{2}$. By applying the operator $\tilde{D}^{\sigma} D^{\tau}$ to (23), we deduce that

$$
\left\|\partial_{t} \tilde{D}_{x}^{\sigma} D_{x}^{\tau} u(t, \cdot)\right\|_{L_{x}^{2}}^{2}+\left\|\tilde{D}_{x}^{\sigma} D_{x}^{\tau+1} u(t, \cdot)\right\|_{L_{x}^{2}}^{2}=\left\|\tilde{D}^{\sigma} D^{\tau} g\right\|_{L^{2}}^{2}+\left\|\tilde{D}^{\sigma} D^{\tau+1} f\right\|_{L^{2}}^{2},
$$

which can be rewritten in terms of Sobolev norms as follows:

$$
\left\|\partial_{t} u(t, \cdot)\right\|_{H^{\sigma, \tau}}^{2}+\|u(t, \cdot)\|_{H^{\sigma, \tau+1}}^{2}=\|g\|_{H^{\sigma, \tau}}^{2}+\|f\|_{H^{\sigma, \tau+1}}^{2} .
$$

\section{Kernel estimates}

In this section we derive pointwise estimates for the radial convolution kernel $w_{t}^{(\sigma, \tau)}$ of the operator $W_{t}^{(\sigma, \tau)}=D^{-\tau} \tilde{D}^{\tau-\sigma} e^{i t D}$, for suitable exponents $\sigma \in \mathbb{R}$ and $\tau \in\left[0, \frac{3}{2}\right)$. To do so, we follow the strategy used in [4] for hyperbolic spaces. The difficulty here is that Damek-Ricci spaces are nonsymmetric in general, so that some of the proofs given in [4] do not work in this context.

By the inversion formula of the spherical Fourier transform,

$$
w_{t}^{(\sigma, \tau)}(r)=\text { const. } \int_{0}^{+\infty} d \lambda|\mathbf{c}(\lambda)|^{-2} \lambda^{-\tau}\left(\lambda^{2}+\frac{\widetilde{Q}^{2}}{4}\right)^{\frac{\tau-\sigma}{2}} \varphi_{\lambda}(r) e^{i t \lambda} .
$$

Let us split up

$$
\begin{aligned}
w_{t}^{(\sigma, \tau)}(r) & =w_{t, 0}^{(\sigma, \tau)}(r)+w_{t, \infty}^{(\sigma, \tau)}(r) \\
& =\text { const. } \int_{0}^{2} d \lambda \chi_{0}(\lambda)|\mathbf{c}(\lambda)|^{-2} \lambda^{-\tau}\left(\lambda^{2}+\frac{\widetilde{Q}^{2}}{4}\right)^{\frac{\tau-\sigma}{2}} \varphi_{\lambda}(r) e^{i t \lambda} \\
& + \text { const. } \int_{1}^{+\infty} d \lambda \chi_{\infty}(\lambda)|\mathbf{c}(\lambda)|^{-2} \lambda^{-\tau}\left(\lambda^{2}+\frac{\widetilde{Q}^{2}}{4}\right)^{\frac{\tau-\sigma}{2}} \varphi_{\lambda}(r) e^{i t \lambda},
\end{aligned}
$$

using smooth cut-off functions $\chi_{0}$ and $\chi_{\infty}$ on $[0,+\infty)$ such that $1=\chi_{0}+\chi_{\infty}, \chi_{0}=1$ on $[0,1]$ and $\chi_{\infty}=1$ on $[2,+\infty)$. We shall first estimate $w_{t, 0}^{(\sigma, \tau)}$ and next a variant of $w_{t, \infty}^{(\sigma, \tau)}$. 
The kernel $w_{t, \infty}^{(\sigma, \tau)}$ has indeed a logarithmic singularity on the sphere $r=t$ when $\sigma=\frac{n+1}{2}$. We bypass this problem by considering the analytic family of operators

$$
\widetilde{W}_{t, \infty}^{(\sigma, \tau)}=\frac{e^{\sigma^{2}}}{\Gamma\left(\frac{n+1}{2}-\sigma\right)} \chi_{\infty}(D) D^{-\tau} \tilde{D}^{\tau-\sigma} e^{i t D}
$$

in the vertical strip $0 \leq \operatorname{Re} \sigma \leq \frac{n+1}{2}$ and the corresponding kernels

$$
\widetilde{w}_{t, \infty}^{(\sigma, \tau)}(r)=\frac{e^{\sigma^{2}}}{\Gamma\left(\frac{n+1}{2}-\sigma\right)} \int_{1}^{+\infty} d \lambda \chi_{\infty}(\lambda)|\mathbf{c}(\lambda)|^{-2} \lambda^{-\tau}\left(\lambda^{2}+\frac{\widetilde{Q}^{2}}{4}\right)^{\frac{\tau-\sigma}{2}} e^{i t \lambda} \varphi_{\lambda}(r) .
$$

Notice that the Gamma function, which occurs naturally in the theory of Riesz distributions, will allow us to deal with the boundary point $\sigma=\frac{n+1}{2}$, while the exponential function yields boundedness at infinity in the vertical strip. Notice also that, once multiplied by $\chi_{\infty}(D)$, the operator $D^{-\tau} \tilde{D}^{\tau-\sigma}$ behaves like $\tilde{D}^{-\sigma}$.

4.1. Estimate of $w_{t}^{0}=w_{t, 0}^{(\sigma, \tau)}$.

Theorem 4.1. Let $\sigma \in \mathbb{R}$ and $\tau<2$. The following pointwise estimates hold for the kernel $w_{t}^{0}=w_{t, 0}^{(\sigma, \tau)}$ :

(i) Assume that $|t| \leq 2$. Then, for every $r \geq 0$,

$$
\left|w_{t}^{0}(r)\right| \lesssim \varphi_{0}(r)
$$

(ii) Assume that $|t| \geq 2$.

(a) If $0 \leq r \leq \frac{|t|}{2}$, then

$$
\left|w_{t}^{0}(r)\right| \lesssim|t|^{\tau-3} \varphi_{0}(r) .
$$

(b) If $r \geq \frac{|t|}{2}$, then

$$
\left|w_{t}^{0}(r)\right| \lesssim(1+|r-| t||)^{\tau-2} e^{-\frac{Q}{2} r} .
$$

Proof. Recall that

$$
w_{t}^{0}(r)=\text { const. } \int_{0}^{2} d \lambda \chi_{0}(\lambda)|\mathbf{c}(\lambda)|^{-2} \lambda^{-\tau}\left(\lambda^{2}+\frac{\widetilde{Q}^{2}}{4}\right)^{\frac{\tau-\sigma}{2}} \varphi_{\lambda}(r) e^{i t \lambda} .
$$

By symmetry we may assume that $t>0$.

(i) It follows from the estimates (10) and (19) that

$$
\left|w_{t}^{0}(r)\right| \lesssim \int_{0}^{2} d \lambda \lambda^{2-\tau} \varphi_{0}(r) \lesssim \varphi_{0}(r) .
$$

(ii) We prove first (a) by substituting the representation (91) of $\varphi_{\lambda}$ in (26). Specifically,

$$
w_{t}^{0}(r)=\text { const. } \int_{\partial B(\mathfrak{s})} d \sigma a(r \sigma)^{Q / 2} \int_{0}^{2} d \lambda \chi_{0}(\lambda) b(\lambda) e^{i\{t-\log a(r \sigma)\} \lambda},
$$

where $b(\lambda)=|\mathbf{c}(\lambda)|^{-2} \lambda^{-\tau}\left(\lambda^{2}+\frac{\widetilde{Q}^{2}}{4}\right)^{\frac{\tau-\sigma}{2}}$ and $a(r \sigma)$ is the $A$-component of the point $r \sigma$ defined in Section 2. According to estimate (7) and to Lemma A.1 in Appendix A, the inner integral is bounded above by

$$
\{t-\log a(r \sigma)\}^{\tau-3} \leq(t-r)^{\tau-3} \asymp t^{\tau-3} \quad \forall \sigma \in \partial B(\mathfrak{s}) .
$$


Since $\pi\left[a(\cdot)^{Q / 2}\right]=\varphi_{0}$, we conclude that

$$
\begin{aligned}
w_{t}^{0}(r) & \lesssim t^{\tau-3} \int_{\partial B(\mathfrak{s})} d \sigma a(r \sigma)^{Q / 2} \\
& =C t^{\tau-3} \pi\left[a(\cdot)^{Q / 2}\right](r) \\
& =C t^{\tau-3} \varphi_{0}(r) .
\end{aligned}
$$

We prove next (b) by substituting in (26) the asymptotic expansion (15) of $\varphi_{\lambda}$ and by reducing to Fourier analysis on $\mathbb{R}$. Specifically,

$$
w_{t}^{0}(r)=\text { const. } e^{-\frac{Q}{2} r} \sum_{\ell=0}^{+\infty} e^{-\ell r}\left\{I_{\ell}^{+, 0}(t, r)+I_{\ell}^{-, 0}(t, r)\right\},
$$

where

and

$$
I_{\ell}^{ \pm, 0}(t, r)=\int_{0}^{2} d \lambda \chi_{0}(\lambda) b_{\ell}^{ \pm}(\lambda) e^{i(t \pm r) \lambda}
$$

$$
b_{\ell}^{ \pm}(\lambda)=\mathbf{c}(\mp \lambda)^{-1} \lambda^{-\tau}\left(\lambda^{2}+\frac{\widetilde{Q}^{2}}{4}\right)^{\frac{\tau-\sigma}{2}} \Gamma_{\ell}( \pm \lambda) .
$$

By applying Lemma A.1 and Lemma 2.1, we obtain

$$
\left|I_{\ell}^{+, 0}(t, r)\right| \lesssim(1+\ell)^{d}(t+r)^{\tau-2} \leq(1+\ell)^{d} r^{\tau-2}
$$

and

$$
\left|I_{\ell}^{-, 0}(t, r)\right| \lesssim(1+\ell)^{d}(1+|r-t|)^{\tau-2},
$$

where $d$ is the constant which appears in Lemma 2.1.

We conclude the proof by summing up these estimates in (27).

4.2. Estimate of $\widetilde{w}_{t}^{\infty}=\widetilde{w}_{t, \infty}^{(\sigma, \tau)}$.

Theorem 4.2. The following pointwise estimates hold for the kernel $\widetilde{w}_{t}^{\infty}=\widetilde{w}_{t, \infty}^{(\sigma, \tau)}$, for any fixed $\tau \in \mathbb{R}$ and uniformly in $\sigma \in \mathbb{C}$ with $\operatorname{Re} \sigma=\frac{n+1}{2}$ :

(i) Assume that $0<|t| \leq 2$.

(a) If $0 \leq r \leq 3$, then $\left|\widetilde{w}_{t}^{\infty}(r)\right| \lesssim|t|^{-\frac{n-1}{2}}$.

(b) If $r \geq 3$, then $\widetilde{w}_{t}^{\infty}(r)=\mathrm{O}\left(r^{-\infty} e^{-\frac{Q}{2} r}\right)$.

(ii) Assume that $|t| \geq 2$. Then

$$
\left|\widetilde{w}_{t}^{\infty}(r)\right| \lesssim(1+|r-| t||)^{-\infty} e^{-\frac{Q}{2} r} \quad \forall r \geq 0 .
$$

Proof of Theorem 4.2. ii. Recall that, up to a positive constant,

$$
\widetilde{w}_{t}^{\infty}(r)=\frac{e^{\sigma^{2}}}{\Gamma\left(\frac{n+1}{2}-\sigma\right)} \int_{1}^{+\infty} d \lambda \chi_{\infty}(\lambda)|\mathbf{c}(\lambda)|^{-2} \lambda^{-\tau}\left(\lambda^{2}+\frac{\widetilde{Q}^{2}}{4}\right)^{\frac{\tau-\sigma}{2}} \varphi_{\lambda}(r) e^{i t \lambda} .
$$

By symmetry we may assume again that $t>0$. If $0 \leq r \leq \frac{t}{2}$, we resume the proof of Theorem 4.1,ii.a, using Lemma A.2 instead of Lemma A.1, and estimate this way

$$
\left|\widetilde{w}_{t}^{\infty}(r)\right| \lesssim(t-r)^{-\infty} \varphi_{0}(r) \lesssim t^{-\infty} e^{-\frac{Q}{2} r}
$$


If $r \geq \frac{t}{2}$, we resume the proof of Theorem 4.1,ii.b and expand this way

$$
\widetilde{w}_{t}^{\infty}(r)=\frac{e^{\sigma^{2}}}{\Gamma\left(\frac{n+1}{2}-\sigma\right)} e^{-\frac{Q}{2} r} \sum_{\ell=0}^{+\infty} e^{-\ell r}\left\{I_{\ell}^{+, \infty}(t, r)+I_{\ell}^{-, \infty}(t, r)\right\}
$$

where

and

$$
I_{\ell}^{ \pm, \infty}(t, r)=\int_{0}^{+\infty} d \lambda \chi_{\infty}(\lambda) b_{\ell}^{ \pm}(\lambda) e^{i(t \pm r) \lambda}
$$

$$
b_{\ell}^{ \pm}(\lambda)=\mathbf{c}(\mp \lambda)^{-1} \lambda^{-\tau}\left(\lambda^{2}+\frac{\widetilde{Q}^{2}}{4}\right)^{\frac{\tau-\sigma}{2}} \Gamma_{\ell}( \pm \lambda) .
$$

It follows from the expression (12) of the c-function and from Lemma 2.1 that $b_{\ell}^{ \pm}$is a symbol of order

$$
\nu= \begin{cases}-1 & \text { if } k=0, \\ -2 & \text { if } k \in \mathbb{N}^{*} .\end{cases}
$$

By Lemma A.2 we obtain that forall $N \in \mathbb{N}^{*}$, there exists a positive constant $C_{N}$ such that for every $\ell \in \mathbb{N}$

$$
\left|I_{\ell}^{+, \infty}(t, r)\right| \leq C_{N}|\sigma|^{N}(1+\ell)^{d}(t+r)^{-N} \leq C_{N}|\sigma|^{N}(1+\ell)^{d} r^{-N},
$$

and for every $\ell \in \mathbb{N}^{*}$

$$
\left|I_{\ell}^{-, \infty}(t, r)\right| \leq C_{N}|\sigma|^{N}(1+\ell)^{d}(1+|r-t|)^{-N},
$$

where $d$ is the constant which appears in Lemma 2.1. To estimate the term $I_{0}^{-, \infty}$ we apply Lemma A.3. To do so, we establish the asymptotic behavior of the symbol $b_{0}^{-}(\lambda)$, as $\lambda \rightarrow+\infty$. On one hand, by (12) we have

$$
\begin{aligned}
\mathbf{c}(\lambda)^{-1} & =\frac{1}{\Gamma\left(\frac{n}{2}\right)} 2^{-Q+i 2 \lambda} \frac{\Gamma\left(i \lambda+\frac{Q}{2}\right) \Gamma\left(i \lambda+\frac{m}{4}+\frac{1}{2}\right)}{\Gamma(i 2 \lambda)} \\
& =C(n, m, Q)\left(\frac{i \lambda+\frac{Q}{2}}{i \lambda}\right)^{i \lambda-\frac{1}{2}}\left(i \lambda+\frac{Q}{2}\right)^{\frac{Q}{2}}\left(\frac{i \lambda+\frac{m}{4}+\frac{1}{2}}{i \lambda}\right)^{i \lambda}\left(i \lambda+\frac{m}{4}+\frac{1}{2}\right)^{\frac{m}{4}}\left\{1+\mathrm{O}\left(\lambda^{-1}\right)\right\} \\
& =C(n, m, Q) \lambda^{\frac{Q}{2}+\frac{m}{4}}\left\{1+\mathrm{O}\left(\lambda^{-1}\right)\right\},
\end{aligned}
$$

according to Stirling's formula

$$
\Gamma(\xi)=\sqrt{2 \pi} \xi^{\xi-\frac{1}{2}} e^{-\xi}\left\{1+\mathrm{O}\left(|\xi|^{-1}\right)\right\} .
$$

On the other hand,

$$
\lambda^{-\tau}\left(\lambda^{2}+\tilde{\rho}^{2}\right)^{\frac{\tau-\sigma}{2}}=\lambda^{-\sigma}\left\{1+\mathrm{O}\left(|\sigma| \lambda^{-2}\right)\right\} .
$$

Since $\frac{Q}{2}+\frac{m}{4}-\operatorname{Re} \sigma=-1$ we get

$$
b_{0}^{-}(\lambda)=c_{0} \lambda^{-1-i \operatorname{Im} \sigma}+r_{0}(\lambda) \text { with }\left|r_{0}(\lambda)\right| \leq C|\sigma| \lambda^{-2} .
$$

As announced, it follows now from Lemma A.3 that

$$
\left|I_{0}^{-, \infty}(t, r)\right| \leq C \frac{|\sigma|^{2}}{|\operatorname{Im} \sigma|} \quad \text { if }|r-t| \leq 1 .
$$

By combining (28), (29), (30), (31) and (32), we conclude that

$$
\left|\widetilde{w}_{t}^{\infty}(r)\right| \lesssim(1+|r-t|)^{-\infty} e^{-\frac{Q}{2} r} \quad \forall r \geq \frac{t}{2} .
$$


The estimate of Theorem 4.2,i.a is of local nature and thus similar to the Euclidean case. For the sake of completeness, we include a proof in Appendix C.

Proof of Theorem 4.2. i.b. Here $0<|t| \leq 2$ and $r \geq 3$. By symmetry we may assume again that $t>0$. Up to positive constants, the inverse spherical Fourier transform (25) can be rewritten in the following way:

$$
\widetilde{w}_{t}^{\infty}(r)=\frac{e^{\sigma^{2}}}{\Gamma\left(\frac{n+1}{2}-\sigma\right)} \mathcal{A}^{-1} g_{t}(r)
$$

where

$$
g_{t}(r)=2 \int_{1}^{+\infty} d \lambda \chi_{\infty}(\lambda) \lambda^{-\tau}\left(\lambda^{2}+\frac{\widetilde{Q}^{2}}{4}\right)^{\frac{\tau-\sigma}{2}} e^{i t \lambda} \cos \lambda r .
$$

Let us split up $2 \cos \lambda r=e^{i \lambda r}+e^{-i \lambda r}$ and $g_{t}(r)=g_{t}^{+}(r)+g_{t}^{-}(r)$ accordingly, so that

$$
g_{t}^{ \pm}(r)=\int_{1}^{+\infty} d \lambda \chi_{\infty}(\lambda) \lambda^{-\tau}\left(\lambda^{2}+\frac{\widetilde{Q}^{2}}{4}\right)^{\frac{\tau-\sigma}{2}} e^{i(t \pm r) \lambda} .
$$

Recall that the inversion formulae (20) and (21) of the Abel transform involve the differential operators $\mathcal{D}_{1}=-\frac{1}{\sinh r} \frac{\partial}{\partial r}$ and $\mathcal{D}_{2}=-\frac{1}{\sinh (r / 2)} \frac{\partial}{\partial r}$. We shall use the fact that, for all integers $p \geq 1$ and $q \geq 1$,

$$
\mathcal{D}_{1}^{p} \mathcal{D}_{2}^{q}=\sum_{j=1}^{p+q} \sum_{\ell=1}^{p} \gamma_{\ell, j}^{\infty}(r)\left(\frac{\partial}{\partial r}\right)^{j},
$$

where the coefficients $\gamma_{\ell, j}^{\infty}(r)$ are linear combinations of products

$$
\begin{aligned}
& \left(\frac{1}{\sinh r}\right) \times\left(\frac{\partial}{\partial r}\right)^{\ell_{2}}\left(\frac{1}{\sinh r}\right) \times \cdots \times\left(\frac{\partial}{\partial r}\right)^{\ell_{m}} \\
& \times\left(\frac{\partial}{\partial r}\right)^{j_{1}}\left(\frac{1}{\sinh (r / 2)}\right) \times \cdots\left(\frac{\partial}{\partial r}\right)^{j_{q}}\left(\frac{1}{\sinh (r / 2)}\right),
\end{aligned}
$$

with $\ell_{2}+\ldots+\ell_{p}=p-\ell$ and $j_{1}+\ldots+j_{q}=q-j+\ell$.

Since $\frac{1}{\sinh r}=2 \sum_{h=0}^{+\infty} e^{-(2 h+1) r}$ is $\mathrm{O}\left(e^{-r}\right)$, as well as its derivatives, we deduce that $\gamma_{\ell, j}^{\infty}(r)$ is $\mathrm{O}\left(e^{-(p+q / 2) r}\right)$ as $r \rightarrow+\infty$. We shall also use the fact that

$$
\left(\frac{\partial}{\partial r}\right)^{j} g_{t}^{ \pm}(r)=\int_{1}^{+\infty} d \lambda \chi_{\infty}(\lambda) \lambda^{-\tau}\left(\lambda^{2}+\frac{\widetilde{Q}^{2}}{4}\right)^{\frac{\tau-\sigma}{2}}( \pm i \lambda)^{j} e^{i(t \pm r) \lambda}
$$

According to Lemma A.2, for every $N \in \mathbb{N}^{*}$, there exists $C_{N} \geq 0$ such that

$$
\left|\left(\frac{\partial}{\partial r}\right)^{j} g_{t}^{ \pm}(r)\right| \leq C_{N}|\sigma|^{N}(r \pm t)^{-N}
$$

- Case 1: Assume that $k$ is even.

By the formula (20) we obtain that

$$
\widetilde{w}_{t}^{\infty}(r)=\text { const. } \frac{e^{\sigma^{2}}}{\Gamma\left(\frac{n+1}{2}-\sigma\right)} \mathcal{D}_{1}^{k / 2} \mathcal{D}_{2}^{m / 2}\left(g_{t}^{+}+g_{t}^{-}\right)(r),
$$

which by (33) and (35) is estimated by

$$
\left|\widetilde{w}_{t}^{\infty}(r)\right| \leq C_{N} r^{-N} e^{-\frac{Q}{2} r} \quad \forall N \in \mathbb{N}^{*} .
$$

- Case 2: Assume that $k$ is odd. 
According to (33) and (35), for every $N \in \mathbb{N}^{*}$, there exists $C_{N} \geq 0$ such that

$$
\left|\mathcal{D}_{1}^{(k+1) / 2} \mathcal{D}_{2}^{m / 2} g_{t}(s)\right| \leq C_{N}|\sigma|^{N} s^{-N} e^{-\frac{Q+1}{2} s} \quad \forall s \geq 3 .
$$

By estimating

$$
\begin{aligned}
& \cosh s-\cosh r=2 \sinh \frac{s+r}{2} \sinh \frac{s-r}{2} \gtrsim e^{r} \sinh \frac{s-r}{2}, \\
& \sinh s \lesssim e^{s}, \quad e^{-\frac{Q}{2} s} \leq e^{-\frac{Q}{2} r}, \quad s^{-N} \leq r^{-N},
\end{aligned}
$$

and performing the change of variables $s=r+u$, we deduce that

$$
\begin{aligned}
\left|\widetilde{w}_{t}^{\infty}(r)\right| & \lesssim \frac{e^{\sigma^{2}}}{\Gamma\left(\frac{n+1}{2}-\sigma\right)} \int_{r}^{+\infty} d s \frac{\sinh s}{\sqrt{\cosh s-\cosh r}}\left|\mathcal{D}_{1}^{(k+1) / 2} \mathcal{D}_{2}^{m / 2} g_{t}(s)\right| \\
& \leq C_{N} \int_{r}^{+\infty} d s \frac{\sinh s}{\sqrt{\cosh s-\cosh r}} s^{-N} e^{-\frac{Q+1}{2} s} \\
& \leq C_{N} r^{-N} e^{-\frac{Q}{2} r} \int_{0}^{+\infty} \frac{d u}{\sqrt{\sinh \frac{u}{2}}} \\
& \leq C_{N} r^{-N} e^{-\frac{Q}{2} r} .
\end{aligned}
$$

\section{Dispersive estimates}

In this section we obtain $L^{q^{\prime}} \rightarrow L^{q}$ estimates for the operator $D^{-\tau} \tilde{D}^{\tau-\sigma} e^{i t D}$, which will be crucial for our Strichartz estimates in next section. Let us split up its kernel $w_{t}=w_{t}^{0}+w_{t}^{\infty}$ as before. We will handle the contribution of $w_{t}^{0}$, using the pointwise estimates obtained in Subsection 4.1 and the following criterion.

Lemma 5.1. There exists a positive constant $C$ such that, for every radial measurable function $\kappa$ on $S$, for every $2 \leq q, \tilde{q}<\infty$ and $f \in L^{q^{\prime}}(S)$,

$$
\|f * \kappa\|_{L^{q}} \leq C\|f\|_{L^{\tilde{q}^{\prime}}}\left\{\int_{0}^{+\infty} d r V(r) \varphi_{0}(r)^{\nu}|\kappa(r)|^{\alpha}\right\}^{\frac{1}{\alpha}} .
$$

where $\nu=\frac{2 \min \{q, \tilde{q}\}}{q+\tilde{q}}, \alpha=\alpha(q, \tilde{q})=\frac{q \tilde{q}}{q+\tilde{q}}$ and $V$ denotes the radial density of the measure $\mu$ as in (8).

Proof. This estimate is obtained by interpolation between the following version of the Herz criterion [17] for Damek-Ricci spaces obtained in [1, Theorem 3.3]

$$
\|f * \kappa\|_{L^{2}} \lesssim\|f\|_{L^{2}} \int_{0}^{+\infty} d r V(r) \varphi_{0}(r)|\kappa(r)|,
$$

and the elementary inequalities

$$
\|f * \kappa\|_{L^{q}} \leq\|f\|_{L^{1}}\|\kappa\|_{L^{q}}, \quad\|f * \kappa\|_{L^{\infty}} \leq\|f\|_{L^{\tilde{q}^{\prime}}}\|\kappa\|_{L^{\tilde{q}}} .
$$

For the second part $w_{t}^{\infty}$, we resume the Euclidean approach, which consists in interpolating analytically between $L^{2} \rightarrow L^{2}$ and $L^{1} \rightarrow L^{\infty}$ estimates for the family of operators

$$
\widetilde{W}_{t, \infty}^{(\sigma, \tau)}=\frac{e^{\sigma^{2}}}{\Gamma\left(\frac{n+1}{2}-\sigma\right)} \chi_{\infty}(D) D^{-\tau} \tilde{D}^{\tau-\sigma} e^{i t D}
$$


in the vertical strip $0 \leq \operatorname{Re} \sigma \leq \frac{n+1}{2}$.

\subsection{Small time dispersive estimate.}

Theorem 5.2. Assume that $0<|t| \leq 2,2<q<\infty, 0 \leq \tau<\frac{3}{2}$ and $\sigma \geq(n+1)\left(\frac{1}{2}-\frac{1}{q}\right)$. Then,

$$
\left\|D^{-\tau} \tilde{D}^{\tau-\sigma} e^{i t D}\right\|_{L^{q^{\prime} \rightarrow L^{q}}} \lesssim|t|^{-(n-1)\left(\frac{1}{2}-\frac{1}{q}\right)} .
$$

Proof. We divide the proof into two parts, corresponding to the kernel decomposition $w_{t}=w_{t}^{0}+w_{t}^{\infty}$. By applying Lemma 5.1 and by using the pointwise estimates in Theorem 4.1.i, we obtain on one hand

$$
\begin{aligned}
\left\|f * w_{t}^{0}\right\|_{L^{q}} & \lesssim\left\{\int_{0}^{+\infty} d r V(r) \varphi_{0}(r)\left|w_{t}^{0}(r)\right|^{\frac{q}{2}}\right\}^{\frac{2}{q}}\|f\|_{L^{q^{\prime}}} \\
& \lesssim\left\{\int_{0}^{+\infty} d r(1+r)^{1+\frac{q}{2}} e^{-\frac{Q}{2} r\left(\frac{q}{2}-1\right)}\right\}^{\frac{2}{q}}\|f\|_{L^{q^{\prime}}} \\
& \lesssim\|f\|_{L^{q^{\prime}}} \quad \forall f \in L^{q^{\prime}} .
\end{aligned}
$$

For the second part, we consider the analytic family (36). If $\operatorname{Re} \sigma=0$, then

$$
\left\|f * \widetilde{w}_{t}^{\infty}\right\|_{L^{2}} \lesssim\|f\|_{L^{2}} \quad \forall f \in L^{2} .
$$

If $\operatorname{Re} \sigma=\frac{n+1}{2}$, we deduce from the pointwise estimates in Theorem 4.2, i that

$$
\left\|f * \widetilde{w}_{t}^{\infty}\right\|_{L^{\infty}} \lesssim|t|^{-\frac{n-1}{2}}\|f\|_{L^{1}} \quad \forall f \in L^{1} .
$$

By interpolation we conclude for $\sigma=(n+1)\left(\frac{1}{2}-\frac{1}{q}\right)$ that

$$
\left\|f * w_{t}^{\infty}\right\|_{L^{q}} \lesssim|t|^{-(n-1)\left(\frac{1}{2}-\frac{1}{q}\right)}\|f\|_{L^{q^{\prime}}} \quad \forall f \in L^{q^{\prime}} .
$$

\subsection{Large time dispersive estimate.}

Theorem 5.3. Assume that $|t| \geq 2,2<q<\infty, 0 \leq \tau<\frac{3}{2}$ and $\sigma \geq(n+1)\left(\frac{1}{2}-\frac{1}{q}\right)$. Then

$$
\left\|D^{-\tau} \tilde{D}^{\tau-\sigma} e^{i t D}\right\|_{L^{q^{\prime} \rightarrow L^{q}}} \lesssim|t|^{\tau-3} \text {. }
$$

Proof. We divide the proof into three parts, corresponding to the kernel decomposition

$$
w_{t}=\mathbb{I}_{B\left(0, \frac{|t|}{2}\right)} w_{t}^{0}+\mathbb{I}_{S \backslash B\left(0, \frac{|t|}{2}\right)} w_{t}^{0}+w_{t}^{\infty} .
$$

Estimate 1: By applying Lemma 5.1 and using the pointwise estimates in Theorem 4.1.ii.a, we obtain

$$
\begin{aligned}
\left\|f *\left\{\mathbb{I}_{B\left(0, \frac{|t|}{2}\right)} w_{t}^{0}\right\}\right\|_{L^{q}} & \lesssim\left\{\int_{0}^{\frac{|t|}{2}} d r V(r) \varphi_{0}(r)\left|w_{t}^{0}(r)\right|^{\frac{q}{2}}\right\}^{\frac{2}{q}}\|f\|_{L^{q^{\prime}}} \\
& \lesssim \underbrace{\left\{\int_{0}^{+\infty} d r(1+r)^{1+\frac{q}{2}} e^{-\frac{Q}{2} r\left(\frac{q}{2}-1\right)}\right\}^{\frac{2}{q}}|t|^{\tau-3}\|f\|_{L^{q^{\prime}}} \quad \forall f \in L^{q^{\prime}} .}_{<+\infty}
\end{aligned}
$$


Estimate 2: By applying Lemma 5.1 and using the pointwise estimates in Theorem 4.1.ii.b, we obtain

$$
\begin{aligned}
\left\|f *\left\{\mathbb{I}_{S \backslash B\left(0, \frac{|t|}{2}\right)} w_{t}^{0}\right\}\right\|_{L^{q}} & \lesssim\left\{\int_{\frac{|t|}{2}}^{+\infty} d r V(r) \varphi_{0}(r)^{\frac{2}{q}}\left|w_{t}^{0}(r)\right|^{\frac{q}{2}}\right\}^{\frac{2}{q}}\|f\|_{L^{q^{\prime}}} \\
& \lesssim \underbrace{\left\{\int_{\frac{|t|}{2}}^{+\infty} d r r e^{-\left(\frac{q}{2}-1\right) \frac{Q}{2} r}\right\}^{\frac{2}{q}}\|f\|_{L^{q^{\prime}}} \quad \forall f \in L^{q^{\prime}} .}_{\lesssim|t|^{-\infty}}
\end{aligned}
$$

Estimate 3: In order to estimate the $L^{q^{\prime}} \rightarrow L^{q}$ norm of $f \mapsto f * w_{t}^{\infty}$, we use interpolation for the analytic family (36). If $\operatorname{Re} \sigma=0$, then

$$
\left\|f * \widetilde{w}_{t}^{\infty}\right\|_{L^{2}} \lesssim\|f\|_{L^{2}} \quad \forall f \in L^{2} .
$$

If $\operatorname{Re} \sigma=\frac{n+1}{2}$, we deduce from Theorem 4.2, ii that

$$
\left\|f * \widetilde{w}_{t}^{\infty}\right\|_{L^{\infty}} \lesssim|t|^{-\infty}\|f\|_{L^{1}} \quad \forall f \in L^{1} .
$$

By interpolation we conclude for $\sigma=(n+1)\left(\frac{1}{2}-\frac{1}{q}\right)$ that

$$
\left\|f * w_{t}^{\infty}\right\|_{L^{q}} \lesssim|t|^{-\infty}\|f\|_{L^{q^{\prime}}} \quad \forall f \in L^{q^{\prime}} .
$$

By taking $\tau=1$ in Theorems 5.2 and 5.3, we obtain in particular the following dispersive estimates.

Corollary 5.4. Let $2<q<\infty$ and $\sigma \geq(n+1)\left(\frac{1}{2}-\frac{1}{q}\right)$. Then

$$
\left\|\tilde{D}^{-\sigma+1} \frac{e^{i t D}}{D}\right\|_{L^{q^{\prime}} \rightarrow L^{q}} \lesssim \begin{cases}|t|^{-(n-1)\left(\frac{1}{2}-\frac{1}{q}\right)} & \text { if } 0<|t| \leq 2 \\ |t|^{-2} & \text { if }|t| \geq 2 .\end{cases}
$$

\section{Strichartz EStimates}

Consider the inhomogeneous linear wave equation on $S$ :

$$
\left\{\begin{array}{l}
\partial_{t}^{2} u(t, x)-\left(\Delta_{S}+\frac{Q^{2}}{4}\right) u(t, x)=F(t, x) \\
u(0, x)=f(x) \\
\left.\partial_{t}\right|_{t=0} u(t, x)=g(x)
\end{array}\right.
$$

whose solution is given by Duhamel's formula:

$$
u(t, x)=\left(\cos t D_{x}\right) f(x)+\frac{\sin t D_{x}}{D_{x}} g(x)+\int_{0}^{t} d s \frac{\sin (t-s) D_{x}}{D_{x}} F(s, x) .
$$

Definition 6.1. A couple $(p, q)$ is called admissible if $\left(\frac{1}{p}, \frac{1}{q}\right)$ belongs to the triangle

$$
T_{n}=\left\{\left(\frac{1}{p}, \frac{1}{q}\right) \in\left(0, \frac{1}{2}\right] \times\left(0, \frac{1}{2}\right) \mid \frac{2}{p}+\frac{n-1}{q} \geq \frac{n-1}{2}\right\} .
$$

From the dispersive estimates obtained above and by arguing as in the proof of Theorem [4, 6.3] we obtain the following result. 
Theorem 6.2. Let $(p, q)$ and $(\tilde{p}, \tilde{q})$ be two admissible couples. Then the following Strichartz estimate holds for solutions to the Cauchy problem (37):

$$
\|u\|_{L^{p}\left(\mathbb{R} ; L^{q}\right)} \lesssim\|f\|_{H^{\sigma-\frac{1}{2}, \frac{1}{2}}}+\|g\|_{H^{\sigma-\frac{1}{2},-\frac{1}{2}}}+\|F\|_{L^{\tilde{p}^{\prime}}\left(\mathbb{R} ; H_{\tilde{q}^{\prime}}^{\sigma+\tilde{\sigma}-1}\right)},
$$

where $\sigma \geq \frac{(n+1)}{2}\left(\frac{1}{2}-\frac{1}{q}\right)$ and $\tilde{\sigma} \geq \frac{(n+1)}{2}\left(\frac{1}{2}-\frac{1}{\tilde{q}}\right)$. Moreover,

$$
\begin{aligned}
& \|u\|_{L^{\infty}\left(\mathbb{R} ; H^{\sigma-\frac{1}{2}, \frac{1}{2}}\right)}+\left\|\partial_{t} u\right\|_{L^{\infty}\left(\mathbb{R} ; H^{\sigma-\frac{1}{2},-\frac{1}{2}}\right)} \\
& \lesssim\|f\|_{H^{\sigma-\frac{1}{2}, \frac{1}{2}}}+\|g\|_{H^{\sigma-\frac{1}{2},-\frac{1}{2}}}+\|F\|_{L^{\tilde{p}^{\prime}}\left(\mathbb{R} ; H_{\tilde{q}^{\prime}}^{\sigma+\tilde{\sigma}-1}\right)} .
\end{aligned}
$$

Remark 6.3. Observe that, in the statement of Theorem [6.2, we may replace $\mathbb{R}$ by any time interval I containing 0 .

\section{GWP RESULTS FOR THE NLW EQUATION ON $S$}

We apply Strichartz estimates for the inhomogeneous linear Cauchy problem associated with the wave equation to prove global well-posedness results for the following nonlinear Cauchy problem

$$
\left\{\begin{array}{l}
\partial_{t}^{2} u(t, x)-\left(\Delta_{S}+\frac{Q^{2}}{4}\right) u(t, x)=F(u(t, x)) \\
u(0, x)=f(x) \\
\left.\partial_{t}\right|_{t=0} u(t, x)=g(x),
\end{array}\right.
$$

with a power-like nonlinearity $F(u)$. By this we mean that

$$
|F(u)| \leq C|u|^{\gamma} \text { and }|F(u)-F(v)| \leq C\left(|u|^{\gamma-1}+|v|^{\gamma-1}\right)|u-v|
$$

for some $C \geq 0$ and $\gamma>1$. Let us recall the definition of global well-posedness.

Definition 7.1. The Cauchy problem (41) is globally well-posed in $H^{\sigma, \tau} \times H^{\sigma, \tau-1}$ if, for any bounded subset $B$ of $H^{\sigma, \tau} \times H^{\sigma, \tau-1}$, there exist a Banach space $X$, continuously embedded into $C\left(\mathbb{R} ; H^{s, \tau}\right) \cap C^{1}\left(\mathbb{R} ; H^{s, \tau-1}\right)$, such that

- for any initial data $(f, g) \in B$, (41) has a unique solution $u \in X$;

- the map $(f, g) \mapsto u$ is continuous from $B$ into $X$.

The amount of smoothness $\sigma$ requested for GWP of (411) in $H^{\sigma-\frac{1}{2}, \frac{1}{2}} \times H^{\sigma-\frac{1}{2},-\frac{1}{2}}$ depends on $\gamma$ and is represented in Figure 1 below. There

$$
\begin{gathered}
\gamma_{1}=\frac{n+3}{n}=1+\frac{3}{n}, \quad \gamma_{2}=\frac{(n+1)^{2}}{(n-1)^{2}+4}=1+\frac{2}{\frac{n-1}{2}+\frac{2}{n-1}}, \quad \gamma_{\text {conf }}=\frac{n+3}{n-1}=1+\frac{4}{n-1}, \\
\gamma_{3}=\frac{n^{2}+5 n-2+\sqrt{n^{4}+2 n^{3}+21 n^{2}-12 n+4}}{2 n^{2}-2 n}=1+\frac{\sqrt{4 n+\left(\frac{n-6}{2}-\frac{2}{n-1}\right)^{2}}-\left(\frac{n-6}{2}-\frac{2}{n-1}\right)}{n}, \\
\gamma_{4}=\frac{n^{2}+2 n-5}{n^{2}-2 n-1}=1+\frac{2}{\frac{n-1}{2}-\frac{1}{n-1}}, \quad \gamma_{\infty}=\min \left\{\gamma_{3}, \gamma_{4}\right\}= \begin{cases}\gamma_{3} & \text { if } n=4,5, \\
\gamma_{4} & \text { if } n \geq 6,\end{cases}
\end{gathered}
$$

and the curves $C_{1}, C_{2}, C_{3}$ are given by

$$
C_{1}(\gamma)=\frac{n+1}{4}\left(1-\frac{n+5}{2 n \gamma-n-1}\right), \quad C_{2}(\gamma)=\frac{n+1}{4}-\frac{1}{\gamma-1}, \quad C_{3}(\gamma)=\frac{n}{2}-\frac{2}{\gamma-1}
$$




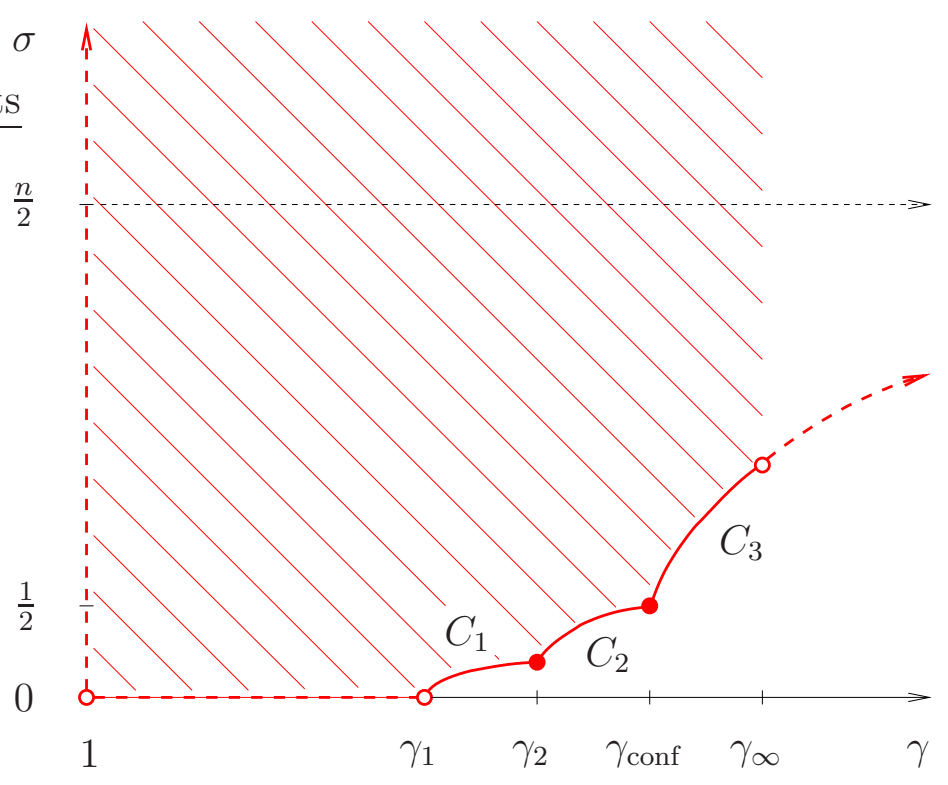

FigURE 1. Regularity in dimension $n \geq 4$

Theorem 7.2. Assume that $F(u)$ satisfies (42). Then (41) is globally well-posed for small initial data in $H^{\sigma-\frac{1}{2}, \frac{1}{2}} \times H^{\sigma-\frac{1}{2},-\frac{1}{2}}$ in the following cases:

(A) $1<\gamma \leq \gamma_{1}$ and $\sigma>0$;

(B) $\gamma_{1}<\gamma \leq \gamma_{2}$ and $\sigma \geq C_{1}(\gamma)$;

(C) $\gamma_{2} \leq \gamma \leq \gamma_{\text {conf }}$ and $\sigma \geq C_{2}(\gamma)$;

(D) $\gamma_{\mathrm{conf}} \leq \gamma<\gamma_{\infty}$ (1) and $\sigma \geq C_{3}(\gamma)$.

More precisely, for such $\gamma$ and $\sigma$, there exists an admissible couple $\left(p_{0}, q_{0}\right)$ and, for sufficiently small initial data $(f, g) \in H^{\sigma-\frac{1}{2}, \frac{1}{2}} \times H^{\sigma-\frac{1}{2},-\frac{1}{2}}$, a unique solution $u$ to (41) such that

$$
u \in C^{1}\left(\mathbb{R} ; H^{\sigma-\frac{1}{2}, \frac{1}{2}}(S)\right) \cap L^{p_{0}}\left(\mathbb{R} ; L^{q_{0}}(S)\right) \quad \text { and } \quad \partial_{t} u \in C\left(\mathbb{R} ; H^{\sigma-\frac{1}{2},-\frac{1}{2}}(S)\right) .
$$

Proof. We apply the standard fixed point method based on Strichartz estimates. Define $u=\Phi(v)$ as the solution to the following linear Cauchy problem

$$
\left\{\begin{array}{l}
\partial_{t}^{2} u(t, x)-D_{x}^{2} u(t, x)=F(v(t, x)), \\
u(0, x)=f(x), \\
\left.\partial_{t}\right|_{t=0} u(t, x)=g(x),
\end{array}\right.
$$

which is given by the Duhamel formula

$$
u(t, x)=\left(\cos t D_{x}\right) f(x)+\frac{\sin t D_{x}}{D_{x}} g(x)+\int_{0}^{t} d s \frac{\sin (t-s) D_{x}}{D_{x}} F(v(s, x)) .
$$

\footnotetext{
${ }^{1}$ The endpoint $\gamma=\gamma_{\infty}$ is excluded in dimension $n=4,5$ and is actually included in dimension $n \geq 6$.
} 
By Theorem 6.2 this solution satisfies the Strichartz estimate

$$
\begin{aligned}
& \|u\|_{L^{\infty}\left(\mathbb{R} ; H^{\sigma-\frac{1}{2}, \frac{1}{2}}\right)}+\left\|\partial_{t} u\right\|_{L^{\infty}\left(\mathbb{R} ; H^{\sigma-\frac{1}{2},-\frac{1}{2}}\right)}+\|u\|_{L^{p}\left(\mathbb{R} ; L^{q}\right)} \\
& \lesssim\|f\|_{H^{\sigma-\frac{1}{2}, \frac{1}{2}}}+\|g\|_{H^{\sigma-\frac{1}{2},-\frac{1}{2}}}+\|F(v)\|_{L^{\tilde{p}^{\prime}}\left(\mathbb{R} ; H_{\tilde{q}^{\prime}}^{\sigma+\tilde{\sigma}-1}\right)},
\end{aligned}
$$

which hold for all admissible couples $(p, q),(\tilde{p}, \tilde{q})$ introduced in Definition 6.1 and for all $\sigma \geq \frac{n+1}{2}\left(\frac{1}{2}-\frac{1}{q}\right), \tilde{\sigma} \geq \frac{n+1}{2}\left(\frac{1}{2}-\frac{1}{\tilde{q}}\right)$. According to the nonlinear assumption (42), we estimate the inhomogeneous term as follows:

$$
\|F(v)\|_{L^{\tilde{p}^{\prime}}\left(\mathbb{R} ; H_{\tilde{q}^{\prime}}^{\sigma+\tilde{\sigma}-1}\right)} \lesssim\left\||v|^{\gamma}\right\|_{L^{\tilde{p}^{\prime}}\left(\mathbb{R} ; H_{\tilde{q}^{\prime}}^{\sigma+\tilde{\sigma}-1}\right)} \cdot
$$

Assuming $\sigma+\tilde{\sigma}-1 \leq n\left(\frac{1}{\tilde{q}^{\prime}}-\frac{1}{\tilde{q}_{1}^{\prime}}\right) \leq 0$, we deduce from Sobolev's embedding (Proposition 3.1) that

$$
\begin{aligned}
& \|u\|_{L^{\infty}\left(\mathbb{R} ; H^{\sigma-\frac{1}{2}, \frac{1}{2}}\right)}+\left\|\partial_{t} u\right\|_{L^{\infty}\left(\mathbb{R} ; H^{\sigma-\frac{1}{2},-\frac{1}{2}}\right)}+\|u\|_{L^{p}\left(\mathbb{R} ; L^{q}\right)} \\
& \lesssim\|f\|_{H^{\sigma-\frac{1}{2}, \frac{1}{2}}}+\|g\|_{H^{\sigma-\frac{1}{2},-\frac{1}{2}}}+\|v\|_{L^{\tilde{p}^{\prime} \gamma}\left(\mathbb{R} ; L^{\tilde{q}_{1}^{\prime} \gamma}\right)}^{\gamma} .
\end{aligned}
$$

In order to remain within the same function space, we require that $q=\tilde{q}_{1}^{\prime} \gamma$ and $p=\tilde{p}^{\prime} \gamma$. It remains for us to check that the following conditions can be fulfilled simultaneously:

$$
\begin{cases}\text { (i) } & p=\tilde{p}^{\prime} \gamma \\ \text { (ii) } & 0<\frac{1}{\tilde{q}^{\prime}} \leq \frac{\gamma}{q}<1 \\ \text { (iii) } & \frac{n-1}{2}-\frac{n+1}{2}\left(\frac{1}{q}+\frac{1}{\tilde{q}}\right) \leq n\left(\frac{1}{\tilde{q}^{\prime}}-\frac{\gamma}{q}\right) \\ \text { (iv) } & \frac{2}{p}+\frac{n-1}{q} \geq \frac{n-1}{2}, \\ \text { (v) } & \frac{2}{\tilde{p}}+\frac{n-1}{\tilde{q}} \geq \frac{n-1}{2} \\ \text { (vi) } & \left(\frac{1}{p}, \frac{1}{q}\right) \in\left(0, \frac{1}{2}\right] \times\left[\frac{n-3}{2(n-1)}, \frac{1}{2}\right) \\ \text { (vii) } & \left(\frac{1}{\tilde{p}}, \frac{1}{\tilde{q}}\right) \in\left(0, \frac{1}{2}\right] \times\left[\frac{n-3}{2(n-1)}, \frac{1}{2}\right)\end{cases}
$$

Suppose indeed that there exist indices $p, q, \tilde{p}, \tilde{q}$ satisfying all conditions in (45). Then (44) shows that $\Phi$ maps $X$ into itself, where $X$ denotes the Banach space

$$
\begin{gathered}
X=\left\{u \mid u \in C\left(\mathbb{R} ; H^{\sigma-\frac{1}{2}, \frac{1}{2}}(S)\right) \cap L^{p}\left(\mathbb{R} ; L^{q}(S)\right),\right. \\
\left.\partial_{t} u \in C\left(\mathbb{R} ; H^{\sigma-\frac{1}{2},-\frac{1}{2}}(S)\right)\right\},
\end{gathered}
$$

equipped with the norm

$$
\|u\|_{X}=\|u\|_{L^{\infty}\left(\mathbb{R} ; H^{\sigma-\frac{1}{2}, \frac{1}{2}}\right)}+\left\|\partial_{t} u\right\|_{L^{\infty}\left(\mathbb{R} ; H^{\sigma-\frac{1}{2},-\frac{1}{2}}\right)}+\|u\|_{L^{p}\left(\mathbb{R} ; L^{q}\right)},
$$

Moreover we shall show that $\Phi$ is a contraction on the ball

$$
X_{\varepsilon}=\left\{u \in X \mid\|u\|_{X} \leq \varepsilon\right\}
$$


provided $\varepsilon>0$ and $\|f\|_{H^{\sigma-\frac{1}{2}, \frac{1}{2}}}+\|g\|_{H^{\sigma-\frac{1}{2},-\frac{1}{2}}}$ are sufficiently small. Let $v, \tilde{v} \in X$ and $u=\Phi(v), \tilde{u}=\Phi(\tilde{v})$. By arguing as above and using Hölder's inequality, we have

$$
\begin{aligned}
\|u-\tilde{u}\|_{X} & \leq C\|F(v)-F(\tilde{v})\|_{L^{\tilde{p}^{\prime}}\left(\mathbb{R} ; H_{\tilde{q}^{\prime}}^{\sigma+\tilde{\sigma}-1}\right)} \\
& \leq C\left\|\left\{|v|^{\gamma-1}+|\tilde{v}|^{\gamma-1}\right\}|v-\tilde{v}|\right\|_{L^{\tilde{p}^{\prime}}\left(\mathbb{R} ; L^{\tilde{q}_{1}^{\prime}}\right)} \\
& \leq C\left\{\|v\|_{L^{p}\left(\mathbb{R} ; L^{q}\right)}^{\gamma-1}+\|\tilde{v}\|_{L^{p}\left(\mathbb{R} ; L^{q}\right)}^{\gamma-1}\right\}\|v-\tilde{v}\|_{L^{p}\left(\mathbb{R} ; L^{q}\right)} \\
& \leq C\left\{\|v\|_{X}^{\gamma-1}+\|\tilde{v}\|_{X}^{\gamma-1}\right\}\|v-\tilde{v}\|_{X} .
\end{aligned}
$$

If $\|v\|_{X} \leq \varepsilon$ and $\|\tilde{v}\|_{X} \leq \varepsilon$ and $\|f\|_{H^{\sigma-\frac{1}{2}, \frac{1}{2}}}+\|g\|_{H^{\sigma-\frac{1}{2},-\frac{1}{2}}} \leq \delta$, then (44) yields on one hand

$$
\|u\|_{X} \leq C \delta+C \varepsilon^{\gamma} \text { and }\|\tilde{u}\|_{X} \leq C \delta+C \varepsilon^{\gamma}
$$

while (46) yields on the other hand

$$
\|u-\tilde{u}\|_{X} \leq 2 C \varepsilon^{\gamma-1}\|v-\tilde{v}\|_{X} .
$$

Thus, if we choose $\varepsilon>0$ and $\delta>0$ so small that $C \varepsilon^{\gamma-1} \leq \frac{1}{4}$ and $C \delta \leq \frac{3}{4} \varepsilon$, then

$$
\|u\|_{X} \leq \varepsilon,\|\tilde{u}\|_{X} \leq \varepsilon \quad \text { and } \quad\|u-\tilde{u}\|_{X} \leq \frac{1}{2}\|v-\tilde{v}\|_{X}
$$

if $v, \tilde{v} \in X_{\varepsilon}$ and $u=\Phi(v), \tilde{u}=\Phi(\tilde{v})$. Hence the map $\Phi$ is a contraction on the complete metric space $X_{\varepsilon}$ and the fixed point theorem allows us to conclude.

Let us eventually prove the existence of couples $(p, q)$ and $(\tilde{p}, \tilde{q})$ satisfying all conditions in (45). Condition (45,iii) amounts to

$$
\frac{2 n \gamma-n-1}{q}+\frac{n-1}{\tilde{q}} \leq n+1 \quad \text { i.e. } \quad \frac{1}{\tilde{q}} \leq \frac{n+1}{n-1}-\frac{2 n \gamma-n-1}{n-1} \frac{1}{q} .
$$

By combining (47) with (45,ii) and (45,vi), we deduce that

$$
\frac{n-3}{2(n-1)} \leq \frac{1}{q} \leq \frac{2}{(\gamma-1)(n+1)} .
$$

This implies that $\gamma \leq \widetilde{\gamma}_{\infty}=\frac{n^{2}+2 n-7}{(n+1)(n-3)}=1+\frac{4(n-1)}{(n+1)(n-3)}$. By combining (47) with (45), vii), we obtain

$$
\frac{n-3}{2(n-1)} \leq \frac{1}{\tilde{q}} \leq \min \left\{\frac{1}{2}, \frac{n+1}{n-1}-\frac{2 n \gamma-n-1}{n-1} \frac{1}{q}\right\}, \quad \frac{1}{\tilde{q}} \neq \frac{1}{2} .
$$

By combining (47) with (45), vii), we also obtain $\frac{1}{q} \leq \frac{n+5}{2(2 n \gamma-n-1)}$. In summary, the conditions on $q$ reduce to

$$
\frac{n-3}{2(n-1)} \leq \frac{1}{q} \leq \min \left\{\frac{1}{2}, \frac{1}{\gamma}, \frac{2}{(\gamma-1)(n+1)}, \frac{n+5}{2(2 n \gamma-n-1)}\right\}, \quad \frac{1}{q} \neq \frac{1}{2}, \frac{1}{\gamma},
$$

or case by case to

- $1<\gamma \leq \gamma_{1}$ and $\frac{n-3}{2(n-1)} \leq \frac{1}{q}<\frac{1}{2}$,

- $\gamma_{1}<\gamma \leq \gamma_{2}$ and $\frac{n-3}{2(n-1)} \leq \frac{1}{q} \leq \frac{n+5}{2(2 n \gamma-n-1)}$,

- $\gamma_{2}<\gamma \leq \widetilde{\gamma}_{\infty}$ and $\frac{n-3}{2(n-1)} \leq \frac{1}{q} \leq \frac{2}{(\gamma-1)(n+1)}$.

Let us turn to the indices $p$ and $\tilde{p}$. According to (45), we have

$$
\frac{n-1}{2}\left(\frac{1}{2}-\frac{1}{q}\right) \leq \frac{1}{p} \leq \frac{1}{2} \quad \text { and } \quad \frac{n-1}{2}\left(\frac{1}{2}-\frac{1}{\tilde{q}}\right) \leq \frac{1}{\tilde{p}} \leq \frac{1}{2} .
$$


Since $\frac{1}{\tilde{p}}=1-\frac{\gamma}{p}$, we end up with the following conditions on $p$ and $\tilde{p}$ :

$$
\left\{\begin{array}{l}
\text { (i) } \quad \frac{n-1}{2}\left(\frac{1}{2}-\frac{1}{q}\right) \leq \frac{1}{p} \leq \min \left\{\frac{1}{2}, \frac{5-n}{4 \gamma}+\frac{n-1}{2 \gamma \tilde{q}}\right\}, \\
\text { (ii) } \frac{n-1}{2}\left(\frac{1}{2}-\frac{1}{\tilde{q}}\right) \leq \frac{1}{\tilde{p}} \leq \frac{1}{2} .
\end{array}\right.
$$

There exist indices $p$ and $\tilde{p}$ which satisfy (48) provided that $\frac{1}{\tilde{q}} \geq \frac{\gamma}{2}+\frac{n-5}{2(n-1)}-\frac{\gamma}{q}$. We thus have to find $\tilde{q}$ such that

$$
\max \left\{\frac{n-3}{2(n-1)}, \frac{\gamma}{2}+\frac{n-5}{2(n-1)}-\frac{\gamma}{q}\right\} \leq \frac{1}{\tilde{q}} \leq \min \left\{\frac{1}{2}, \frac{n+1}{n-1}-\frac{2 n \gamma-n-1}{(n-1) q}\right\},
$$

with $\frac{1}{\tilde{q}} \neq \frac{1}{2}$. This implies that $q$ has to satisfy the following conditions:

$$
\max \left\{\frac{n-3}{2(n-1)}, \frac{1}{2}-\frac{2}{\gamma(n-1)}\right\} \leq \frac{1}{q} \leq \min \left\{\frac{1}{2}, \frac{1}{\gamma}, \frac{2}{(\gamma-1)(n+1)}, \frac{n+5}{2(2 n \gamma-n-1)}, \frac{n+7-\gamma(n-1)}{2(\gamma-1)(n+1)}\right\},
$$

with $\frac{1}{q} \neq \frac{1}{2}-\frac{2}{\gamma(n-1)}, \frac{1}{2}, \frac{1}{\gamma}$. The fact that $\frac{n-3}{2(n-1)} \leq \frac{n+7-\gamma(n-1)}{2(\gamma-1)(n+1)}$ easily implies that $\gamma \leq \gamma_{4}<$ $\tilde{\gamma}_{\infty}$. The fact that $\frac{1}{2}-\frac{2}{\gamma(n-1)}<\frac{n+7-\gamma(n-1)}{2(\gamma-1)(n+1)}$ implies that $\gamma<\gamma_{3}$. In summary, here are the final conditions on $q$, depending on $\gamma$ and possibly on the dimension $n$ :

(A) $1<\gamma \leq \gamma_{1}=1+\frac{3}{n}$ and $\frac{n-3}{2(n-1)} \leq \frac{1}{q}<\frac{1}{2}$.

(B) $\gamma_{1}<\gamma \leq \gamma_{2}=\frac{(n+1)^{2}}{n^{2}-2 n+5}$ and $\frac{n-3}{2(n-1)} \leq \frac{1}{q} \leq \frac{n+5}{2(2 n \gamma-n-1)}$.

(C) $\gamma_{2}<\gamma<\gamma_{\text {conf }}$ and $\frac{n-3}{2(n-1)} \leq \frac{1}{q} \leq \frac{2}{(\gamma-1)(n+1)}$ when $n \geq 5$.

When $n=4$, we distinguish two subcases:

- $\gamma_{2}<\gamma \leq 2$ and $\frac{n-3}{2(n-1)} \leq \frac{1}{q} \leq \frac{2}{(\gamma-1)(n+1)}$,

- $2<\gamma<\gamma_{\text {conf }}$ and $\frac{1}{2}-\frac{2}{\gamma(n-1)}<\frac{1}{q} \leq \frac{2}{(\gamma-1)(n+1)}$.

(D) When $n \geq 6$, we distinguish two subcases:

- $\gamma_{\text {conf }} \leq \gamma \leq 2$ and $\frac{n-3}{2(n-1)} \leq \frac{1}{q} \leq \frac{n+7-\gamma(n-1)}{2(\gamma-1)(n+1)}$,

- $2<\gamma \leq \gamma_{4}$ and $\frac{1}{2}-\frac{2}{\gamma(n-1)}<\frac{1}{q} \leq \frac{n+7-\gamma(n-1)}{2(\gamma-1)(n+1)}$.

When $n=5$, we replace $\gamma_{4}$ by $\gamma_{3}$ and require $\gamma<\gamma_{3}$.

When $n=4, \gamma_{\text {conf }} \leq \gamma<\gamma_{3}$ and $\frac{1}{2}-\frac{2}{\gamma(n-1)}<\frac{1}{q} \leq \frac{n+7-\gamma(n-1)}{2(\gamma-1)(n+1)}$.

Let us now examine these cases separately.

Case (A). In this case, we choose successively $q$ such that

$$
\frac{n-3}{2(n-1)} \leq \frac{1}{q}<\frac{1}{2}
$$

$\tilde{q}$ satisfying (49), and $p, \tilde{p}$ satisfying (48). Thus, when $1<\gamma \leq \gamma_{1}$ and $\sigma>0$, there exists always an admissible couple $(p, q)$ such that all conditions (45) are satisfied and $\sigma \geq \frac{(n+1)}{2}\left(\frac{1}{2}-\frac{1}{q}\right)$.

Case (B). In this case, we choose successively $q$ such that

$$
\frac{n-3}{2(n-1)} \leq \frac{1}{q} \leq \frac{n+5}{2(2 n \gamma-n-1)}
$$

$p, \tilde{p}$ satisfying (48), and a correspondent $\tilde{q}$ which satisfies (49). Thus, when $\gamma_{1}<\gamma \leq \gamma_{2}$ and $\sigma \geq \frac{n+1}{4}-\frac{(n+1)(n+5)}{4(2 n \gamma-n-1)}$, there exists an admissible couple $(p, q)$ such that all conditions (45)) are satisfied and $\sigma \geq \frac{(n+1)}{2}\left(\frac{1}{2}-\frac{1}{q}\right)$. 
Case (C). Assume first that $n \geq 5$. we choose successively $q$ such that

$$
\frac{n-3}{2(n-1)} \leq \frac{1}{q} \leq \frac{2}{(\gamma-1)(n+1)},
$$

$\tilde{q}$ satisfying (49), and $p, \tilde{p}$ satisfying (48).

Assume next that $n=4$. If $\gamma_{2}<\gamma \leq 2$, we choose $q$ according to (51). If $2<\gamma<\gamma_{\text {conf }}$, we replace (51) by

$$
\frac{1}{2}-\frac{2}{\gamma(n-1)}<\frac{1}{q} \leq \frac{2}{(\gamma-1)(n+1)} .
$$

In both cases, we can choose afterwards $\tilde{q}, p, \tilde{p}$ satisfying (49) and (48).

In summary, when $\gamma_{2}<\gamma<\gamma_{\text {conf }}$ and $\sigma \geq \frac{n+1}{4}-\frac{1}{\gamma-1}$, there exists always an admissible couple $(p, q)$ such that all conditions (45) are satisfied and $\sigma \geq \frac{(n+1)}{2}\left(\frac{1}{2}-\frac{1}{q}\right)$.

Case (D). Assume first that $n \geq 6$. If $\gamma_{\mathrm{conf}} \leq \gamma \leq 2$, we choose successively $q$ such that

$$
\frac{n-3}{2(n-1)} \leq \frac{1}{q} \leq \frac{n+7-\gamma(n-1)}{2(\gamma-1)(n+1)}
$$

$\tilde{q}$ satisfying (49), and $p, \tilde{p}$ satisfying (48). If $2<\gamma \leq \gamma_{4}$, (52) is replaced by

$$
\frac{1}{2}-\frac{2}{\gamma(n-1)}<\frac{1}{q} \leq \frac{n+7-\gamma(n-1)}{2(\gamma-1)(n+1)} .
$$

Assume next that $n=5$. We choose again $q$ according to (52) if $\gamma_{\text {conf }} \leq \gamma \leq 2$ and according to (53) if $2<\gamma<\gamma_{3}$. In both cases, we can choose afterwards $\tilde{q}, p, \tilde{p}$ satisfying (49) and (48).

Assume eventually that $n=4$. Then we choose $q$ according to (52) and $\tilde{q}, p, \tilde{p}$ satisfying (49) and (48).

In summary, in this case when $\sigma \geq \frac{n}{2}-\frac{2}{\gamma-1}$, there exists always an admissible couple $(p, q)$ such that all conditions (45) are satisfied and $\sigma \geq \frac{n+1}{2}\left(\frac{1}{2}-\frac{1}{q}\right)$.

This concludes the proof of Theorem 7.2 .

\section{Appendix A}

In this appendix we collect some lemmata in Fourier analysis on $\mathbb{R}$ which are used for the kernel analysis in Section 4 and in Appendix C. These lemmata are proved in [4, Appendix A].

Lemma A.1. Let $b$ be a compactly supported homogeneous symbol on $\mathbb{R}$ of order $\nu>-1$. In other words, $b$ is a smooth function on $\mathbb{R}^{*}$, whose support is bounded in $\mathbb{R}$ and which has the following behavior at the origin:

$$
\sup _{\lambda \in \mathbb{R}^{*}}|\lambda|^{\ell-\nu}\left|\partial_{\lambda}^{\ell} b(\lambda)\right|<+\infty \quad \forall \ell \in \mathbb{N} .
$$

Then its Fourier transform

$$
k(x)=\int_{0}^{+\infty} d \lambda b(\lambda) e^{i \lambda x}
$$

is a smooth function on $\mathbb{R}$, with the following behavior at infinity:

$$
k(x)=\mathrm{O}\left(|x|^{-\nu-1}\right) \quad \text { as }|x| \rightarrow \infty .
$$


More precisely, let $N$ be the smallest integer $>d+1$. Then $\exists C \geq 0, \forall x \in \mathbb{R}^{*}$,

$$
|k(x)| \leq C|x|^{-\nu-1} \sum_{\ell=0}^{N} \sup _{\lambda \in \mathbb{R}^{*}}(1+|\lambda|)^{\ell-\nu}\left|\partial_{\lambda}^{\ell} b(\lambda)\right| .
$$

Lemma A.2. Let $b$ be an inhomogeneous symbol on $\mathbb{R}$ of order $\nu \in \mathbb{R}$. In other words, $b$ is a smooth function on $\mathbb{R}$ such that

$$
\sup _{\lambda \in \mathbb{R}}(1+|\lambda|)^{\ell-\nu}\left|\partial_{\lambda}^{\ell} b(\lambda)\right|<+\infty \quad \forall \ell \in \mathbb{N} .
$$

Then its Fourier transform

$$
k(x)=\int_{-\infty}^{+\infty} d \lambda b(\lambda) e^{i \lambda x}
$$

is a smooth function on $\mathbb{R}^{*}$, which has the following asymptotic behaviors:

(i) At infinity, $k(x)=\mathrm{O}\left(|x|^{-\infty}\right)$. More precisely, for every $N>\nu+1$, there exists $C_{N} \geq 0$ such that, for every $x \in \mathbb{R}^{*}$,

$$
|k(x)| \leq C_{N}|x|^{-N} \sup _{\lambda \in \mathbb{R}}(1+|\lambda|)^{N-\nu}\left|\partial_{\lambda}^{N} b(\lambda)\right| .
$$

(ii) At the origin,

$$
k(x)= \begin{cases}\mathrm{O}(1) & \text { if } \nu<-1 \\ \mathrm{O}\left(\log \frac{1}{|x|}\right) & \text { if } \nu=-1 \\ \mathrm{O}\left(|x|^{-\nu-1}\right) & \text { if } \nu>-1\end{cases}
$$

More precisely:

- If $\nu<-1$, then there exists $C \geq 0$ such that, for every $x \in \mathbb{R}$,

$$
|k(x)| \leq C \sup _{\lambda \in \mathbb{R}}(1+|\lambda|)^{-\nu}|b(\lambda)| .
$$

○ If $\nu=-1$, then there exists $C \geq 0$ such that, for every $0<|x|<\frac{1}{2}$,

$$
|k(x)| \leq C \log \frac{1}{|x|}\left\{\sup _{\lambda \in \mathbb{R}}(1+|\lambda|)|b(\lambda)|+\sup _{\lambda \in \mathbb{R}}(1+|\lambda|)^{2}\left|b^{\prime}(\lambda)\right|\right\} .
$$

- If $\nu>-1$, let $N$ be the smallest integer $>\nu+1$. Then there exists $C \geq 0$ such that, for every $0<|x|<1$,

$$
|k(x)| \leq C|x|^{-\nu-1} \sum_{\ell=0}^{N} \sup _{\lambda \in \mathbb{R}}(1+|\lambda|)^{\ell-\nu}\left|\partial_{\lambda}^{\ell} b(\lambda)\right| .
$$

(iii) Similar estimates hold for the derivatives

$$
\partial_{x}^{\ell} k(x)=\int_{-\infty}^{+\infty} d \lambda(i \lambda)^{\ell} b(\lambda) e^{i \lambda x}
$$

which correspond to symbols $b_{\ell}(\lambda)=(i \lambda)^{\ell} a(\lambda)$ of order $\nu+\ell$. 
Lemma A.3. Assume that

$$
b(\lambda)=\zeta \chi_{\infty}(\lambda) \lambda^{-m-1-i \zeta}+f(\lambda)
$$

where $m \in \mathbb{N}, \zeta \in \mathbb{R}$, and $f$ is a symbol of order $\nu<-m-1$. Then

$$
\partial_{x}^{m} k(x)=\int_{-\infty}^{+\infty} d \lambda b(\lambda)(i \lambda)^{m} e^{i \lambda x}
$$

is a bounded function at the origin. More precisely, there exists $C \geq 0$ such that, for every $0<|x|<\frac{1}{2}$,

$$
\left|\partial_{x}^{m} k(x)\right| \leq C\left\{1+\zeta^{2}+\sup _{\lambda \in \mathbb{R}}(1+|\lambda|)^{-\nu}|f(\lambda)|\right\}
$$

\section{Appendix B}

In this appendix we collect some properties of the Riesz distributions. We refer to 13 , ch. $1, \S 3$ \& ch. $2, \S 2]$ or [18, ch. III, $\S 3.2]$ for more details. The Riesz distribution $R_{z}^{+}$ is defined by

$$
\left\langle R_{z}^{+}, \varphi\right\rangle=\frac{1}{\Gamma(z)} \int_{0}^{+\infty} d \lambda \lambda^{z-1} \varphi(\lambda)
$$

when $\operatorname{Re} z>0$. It extends to a holomorphic family $\left\{R_{z}^{+}\right\}_{z \in \mathbb{C}}$ of tempered distributions on $\mathbb{R}$ which satisfy the following properties:

(i) $\lambda R_{z}^{+}=z R_{z+1}^{+} \quad \forall z \in \mathbb{C}$,

(ii) $\left(\frac{d}{d \lambda}\right) R_{z}^{+}=R_{z-1}^{+} \forall z \in \mathbb{C}$,

(iii) $R_{0}^{+}=\delta_{0}$ and more generally $R_{-m}^{+}=\left(\frac{d}{d \lambda}\right)^{m} \delta_{0} \forall m \in \mathbb{N}$,

(iv) $R_{z+z^{\prime}}^{+}=R_{z}^{+} * R_{z^{\prime}}^{+} \forall z, z^{\prime} \in \mathbb{C}$.

Hence

$$
\left\langle R_{z}^{+}, \varphi\right\rangle=\left\langle\left(\frac{d}{d \lambda}\right)^{m} R_{z+m}^{+}, \varphi\right\rangle=\frac{(-1)^{m}}{\Gamma(z+m)} \int_{0}^{+\infty} d \lambda \lambda^{z+m-1}\left(\frac{d}{d \lambda}\right)^{m} \varphi(\lambda)
$$

when $\operatorname{Re} z>-m$. The Riesz distribution $R_{z}^{-}=\left(R_{z}^{+}\right)^{\vee}$ is defined similarly. Their Fourier transforms are given by

(v) $\mathcal{F} R_{z}^{ \pm}=e^{ \pm i \frac{\pi}{2} z}(x \pm i 0)^{-z} \forall z \in \mathbb{C}$,

where

$$
\left\langle(x \pm i 0)^{z}, \varphi\right\rangle=\lim _{\varepsilon \searrow 0} \int_{\mathbb{R}} d x(x \pm i \varepsilon)^{z} \varphi(x)
$$

when $\operatorname{Re} z>-1$ and

$$
(x \pm i 0)^{z}=\Gamma(z+1)\left\{R_{z+1}^{+}+e^{ \pm i \pi z} R_{z+1}^{-}\right\}
$$

in general (notice that there are actually no singularities in the last expression). 


\section{Appendix C}

In this appendix we prove the local kernel estimates

$$
\left|\widetilde{w}_{t}^{\infty}(r)\right| \lesssim|t|^{-\frac{n-1}{2}}
$$

stated in Theorem 4.2. i.a under the assumptions $0<|t| \leq 2,0 \leq r \leq 3$ and $\operatorname{Re} \sigma=\frac{n+1}{2}$. By symmetry, we may assume again that $t>0$.

- Case 1: Assume that $r \leq \frac{t}{2}$.

By using the representation (9) of the spherical functions, we obtain

$$
\widetilde{w}_{t}^{\infty}(r)=\frac{e^{\sigma^{2}}}{\Gamma(-i \operatorname{Im} \sigma)} \int_{\partial B(\mathfrak{s})} d \sigma a^{\frac{Q}{2}}(r \sigma) \int_{1}^{\infty} d \lambda \chi_{\infty}(\lambda) b(\lambda) e^{i \lambda\{t-\log a(r \sigma)\}},
$$

where

$$
b(\lambda)=|\mathbf{c}(\lambda)|^{-2} \lambda^{-\tau}\left(\lambda^{2}+\frac{\widetilde{Q}^{2}}{4}\right)^{\frac{\tau-\sigma}{2}},
$$

and $a(r \sigma)$ is the $A$-component of the point $r \sigma$. By (7)

$$
|t-\log a(r \sigma)| \geq t-r \geq \frac{t}{2} \quad \forall \sigma \in \partial B(\mathfrak{s}),
$$

so that according to Lemma A.2 in Appendix A, since $\chi_{\infty} b$ is a symbol of order $\frac{n-3}{2}$ the inner integral in (56) is

$$
\mathrm{O}\left(|\sigma|^{N}|t-\log a(r \sigma)|^{-\frac{n-1}{2}}\right)=\mathrm{O}\left(|\sigma|^{N} t^{-\frac{n-1}{2}}\right),
$$

where $N$ is the smallest integer $>\frac{n-1}{2}$. Hence

- Case 2: Assume that $r>\frac{t}{2}$.

$$
\left|\widetilde{w}_{t}^{\infty}(r)\right| \lesssim t^{-\frac{n-1}{2}}
$$

In this case we estimate $\widetilde{w}_{t}(r)$ using the inverse Abel transform. More precisely, we apply the inversion formulae (20) and (21) to the Euclidean Fourier transform

$$
\widetilde{g}_{t}^{\infty}(r)=\frac{e^{\sigma^{2}}}{\Gamma(-i \operatorname{Im} \sigma)} \int_{1}^{+\infty} d \lambda \chi_{\infty}(\lambda)|\mathbf{c}(\lambda)|^{-2} \lambda^{-\tau}\left(\lambda^{2}+\frac{\widetilde{Q}^{2}}{4}\right)^{\frac{\tau-\sigma}{2}} e^{i t \lambda} \cos \lambda r .
$$

We shall use the fact that, for all integers $p \geq 1$ and $q \geq 1$,

$$
\mathcal{D}_{1}^{p} \mathcal{D}_{2}^{q}=\sum_{j=1}^{p+q} \sum_{\ell=1}^{p} \gamma_{\ell, j}^{0}(r)\left(\frac{1}{r} \frac{\partial}{\partial r}\right)^{j},
$$

where the coefficients $\gamma_{\ell, j}^{0}(r)$ in (57) are smooth functions on $\mathbb{R}$, which are linear combinations of products

$$
\begin{aligned}
& \left(\frac{r}{\sinh r}\right) \times\left(\frac{1}{r} \frac{\partial}{\partial r}\right)^{\ell_{2}}\left(\frac{r}{\sinh r}\right) \times \cdots \times\left(\frac{1}{r} \frac{\partial}{\partial r}\right)^{\ell_{p}}\left(\frac{r}{\sinh r}\right) \\
& \times\left(\frac{1}{r} \frac{\partial}{\partial r}\right)^{j_{1}}\left(\frac{r}{\sinh (r / 2)}\right) \times \cdots \times\left(\frac{1}{r} \frac{\partial}{\partial r}\right)^{j_{q}}\left(\frac{r}{\sinh (r / 2)}\right)
\end{aligned}
$$

with $\ell_{2}+\ldots+\ell_{p}=p-\ell$ and $j_{1}+\ldots+j_{q}=q-j+\ell$. We shall also use the following expansion

$$
\left(\frac{1}{r} \frac{\partial}{\partial r}\right)^{j}=\sum_{h=1}^{j} \beta_{j, h} r^{h-2 j}\left(\frac{\partial}{\partial r}\right)^{h}
$$


where the coefficients $\beta_{j, h}$ in (58) are constants.

- Subcase 2.a: Assume that $k$ is even. Then, up to a multiplicative constant,

$$
\widetilde{w}_{t}^{\infty}(r)=\mathcal{D}_{1}^{k / 2} \mathcal{D}_{2}^{m / 2} \widetilde{g}_{t}^{\infty}(r) .
$$

Consider first

$$
\frac{e^{\sigma^{2}}}{\Gamma(-i \operatorname{Im} \sigma)} \int_{1}^{\frac{6}{r}} d \lambda \chi_{\infty}(\lambda) \lambda^{-\tau}\left(\lambda^{2}+\frac{\widetilde{Q}^{2}}{4}\right)^{\frac{\tau-\sigma}{2}} e^{i t \lambda}\left(\frac{1}{r} \frac{\partial}{\partial r}\right)^{j} \cos \lambda r .
$$

Since $\chi_{\infty}(\lambda) \lambda^{-\tau}\left(\lambda^{2}+\frac{\widetilde{Q}^{2}}{4}\right)^{\frac{\tau-\sigma}{2}} e^{i t \lambda}=\mathrm{O}\left(\lambda^{-\frac{n+1}{2}}\right)$ according to the assumption $\operatorname{Re} \sigma=\frac{n+1}{2}$, and $\left(\frac{1}{r} \frac{\partial}{\partial r}\right)^{j} \cos \lambda r=\mathrm{O}\left(\lambda^{2 j}\right)$ by Taylor's formula, the expression (59) is

$$
\begin{cases}\mathrm{O}(1) & \text { if } 1 \leq j<\frac{n-1}{4} \\ \mathrm{O}\left(\log \frac{1}{r}\right) & \text { if } j=\frac{n-1}{4} \\ \mathrm{O}\left(r^{\frac{n-1}{2}}-2 j\right) & \text { if } \frac{n-1}{4}<j \leq \frac{n-1}{2}\end{cases}
$$

hence $\mathrm{O}\left(r^{-\frac{n-1}{2}}\right)$ in all cases. Consider next

$$
\frac{e^{\sigma^{2}}}{\Gamma(-i \operatorname{Im} \sigma)} \int_{\frac{6}{r}}^{+\infty} d \lambda \lambda^{-\tau}\left(\lambda^{2}+\frac{\widetilde{Q}^{2}}{4}\right)^{\frac{\tau-\sigma}{2}} r^{h-2 j}\left(\frac{\partial}{\partial r}\right)^{h} e^{i(t \pm r) \lambda} .
$$

Since $\left(\frac{\partial}{\partial r}\right)^{h} e^{i(t \pm r) \lambda}=( \pm i \lambda)^{h} e^{i(t \pm r) \lambda}$ and

$$
\lambda^{-\tau}\left(\lambda^{2}+\frac{\widetilde{Q}^{2}}{4}\right)^{\frac{\tau-\sigma}{2}}( \pm i \lambda)^{h} e^{i(t \pm r) \lambda}=\mathrm{O}\left(\lambda^{h-\frac{n+1}{2}}\right),
$$

the expression (60) is easily seen to be $\mathrm{O}\left(r^{\frac{n-1}{2}-2 j}\right)$ as long as $h<\frac{n-1}{2}$. For the remaining case, where $h=j=\frac{n-1}{2}$, let us expand

$$
\lambda^{-\tau}\left(\lambda^{2}+\tilde{\rho}^{2}\right)^{\frac{\tau-\sigma}{2}} \lambda^{\frac{n-1}{2}}=\lambda^{-1-i \operatorname{Im} \sigma}\left(1+\frac{\widetilde{Q}^{2}}{4 \lambda^{2}}\right)^{\frac{\tau-\sigma}{2}}=\lambda^{-1-i \operatorname{Im} \sigma}+\mathrm{O}\left(|\sigma| \lambda^{-3}\right)
$$

and split

$$
\int_{\frac{6}{r}}^{+\infty}=\int_{\frac{6}{r}}^{\frac{6}{r}+\frac{1}{|r \pm t|}}+\int_{\frac{6}{r}+\frac{1}{|r \pm t|}}^{+\infty}
$$

in (60). The previous splitting is meaningful only if $r \neq t$. On one hand, the resulting integrals

$$
I_{ \pm}=\frac{e^{\sigma^{2}}}{\Gamma(-i \operatorname{Im} \sigma)} \int_{\frac{6}{r}}^{\frac{6}{r}+\frac{1}{|r \pm t|}} d \lambda \lambda^{-1-i \operatorname{Im} \sigma} e^{i(t \pm r) \lambda}
$$

and

$$
I I_{ \pm}=\frac{e^{\sigma^{2}}}{\Gamma(-i \operatorname{Im} \sigma)} \int_{\frac{6}{r}+\frac{1}{|r \pm t|}}^{+\infty} d \lambda \lambda^{-1-i \operatorname{Im} \sigma} e^{i(t \pm r) \lambda}
$$

are uniformly bounded. This is proved by integrations by parts: 


$$
\begin{gathered}
I_{ \pm}=\frac{e^{\sigma^{2}}}{\Gamma(1-i \operatorname{Im} \sigma)} \overbrace{\left.\lambda^{-i \operatorname{Im} \sigma} e^{i(t \pm r) \lambda}\right|_{\lambda=\frac{6}{r}} ^{\lambda=\frac{6}{r}+\frac{1}{r \pm t \mid}}}^{\mathrm{O}(1)} \\
\mp i \frac{e^{\sigma^{2}}}{\Gamma(1-i \operatorname{Im} \sigma)}(r \pm t) \underbrace{\int_{\frac{1}{r}}^{\frac{1}{r}+\frac{1}{r \pm t \mid}} d \lambda \lambda^{-i \operatorname{Im} \sigma} e^{i(t \pm r) \lambda}}_{\mathrm{O}\left(\frac{1}{|r \pm t|}\right)}=\mathrm{O}(1),
\end{gathered}
$$

while

$$
\begin{aligned}
& I_{ \pm}=\mp i \frac{e^{\sigma^{2}}}{\Gamma(-i \operatorname{Im} \sigma)} \frac{1}{r \pm t} \overbrace{\left.\lambda^{-1-i \operatorname{Im} \sigma} e^{i(t \pm r) \lambda}\right|_{\lambda=\frac{6}{r}+\frac{1}{r \pm t}} ^{\lambda=+\infty}}^{\mathrm{O}(|r \pm t|)} \\
& \mp i \frac{e^{\sigma^{2}}(1+i \operatorname{Im} \sigma)}{\Gamma(-i \operatorname{Im} \sigma)} \frac{1}{r \pm t} \underbrace{\int_{\frac{6}{r}+\frac{1}{r \pm t}}^{+\infty} d \lambda \lambda^{-2-i \operatorname{Im} \sigma} e^{i(t \pm r) \lambda}}_{\mathrm{O}(|r \pm t|)}=\mathrm{O}(1) .
\end{aligned}
$$

Hence the contributions of (61) and (62) to (60) are $\mathrm{O}\left(r^{-\frac{n-1}{2}}\right)$. On the other hand, the remainder's contribution to (60) is obviously $\mathrm{O}\left(r^{2-\frac{n-1}{2}}\right)$. As a conclusion, for all $r>\frac{t}{2}$ and $r \neq t$,

$$
\left|\widetilde{w}_{t}^{\infty}(r)\right| \lesssim r^{-\frac{n-1}{2}} \lesssim t^{-\frac{n-1}{2}}
$$

If $r=t$, the estimates follows as before and is even easier, because $e^{i(t-r) \lambda}=1$.

- Subcase 2.b: Assume that $k$ is odd. Then, up to a multiplicative constant,

$$
\widetilde{w}_{t}^{\infty}(r)=\frac{e^{\sigma^{2}}}{\Gamma(-i \operatorname{Im} \sigma)} \int_{r}^{+\infty} d s \frac{\sinh s}{\sqrt{\cosh s-\cosh r}} \mathcal{D}_{1}^{(k+1) / 2} \mathcal{D}_{2}^{m / 2} \widetilde{g}_{t}^{\infty}(s) .
$$

Let us split

$$
\int_{r}^{+\infty}=\int_{r}^{6}+\int_{6}^{+\infty}
$$

The following estimate is obtained by resuming the proof of Theorem 4.2.i.b in the odd-dimensional case :

$$
\left|\mathcal{D}_{1}^{(k+1) / 2} \mathcal{D}_{2}^{m / 2} \widetilde{g}_{t}^{\infty}(s)\right| \lesssim e^{-\frac{Q+1}{2} s} \quad \forall s \geq 6
$$

Since

$$
\int_{6}^{+\infty} d s \frac{\sinh s}{\sqrt{\cosh s-\cosh r}} e^{-\frac{Q+1}{2} s} \lesssim \int_{0}^{+\infty} \frac{d u}{\sqrt{\sinh u}}<+\infty,
$$

the contribution to (63) of the second integral in (64) is uniformly bounded. Thus we are left with the contribution of the first integral, which is a purely local estimate. To do so, we argue as in [4, Lemma C.1] and obtain the following result.

Lemma C.1 Let $p, q$ be two integers $\geq 1$ and let $\lambda \geq 1, r \leq 3$. 
(i) Assume that $\lambda r \leq 6$. Then

$$
\theta(\lambda, r)=\int_{r}^{6} d s \frac{\sinh s}{\sqrt{\cosh s-\cosh r}} \mathcal{D}_{1}^{p} \mathcal{D}_{2}^{q} \cos \lambda s
$$

is $\mathrm{O}\left(\lambda^{2 p+2 q-1-\varepsilon} r^{-\varepsilon}\right)$, for every $\varepsilon>0$.

(ii) Assume that $\lambda r \geq 6$. Then

$$
\theta^{ \pm}(\lambda, r)=\int_{r}^{6} d s \frac{\sinh s}{\sqrt{\cosh s-\cosh r}} \mathcal{D}_{1}^{p} \mathcal{D}_{2}^{q} e^{ \pm i \lambda s}
$$

has the following behavior:

$$
\theta^{ \pm}(\lambda, r)=c_{ \pm} \lambda^{p+q-\frac{1}{2}}(\sinh r)^{\frac{1}{2}-p-q} e^{ \pm i \lambda r}+\mathrm{O}\left(\lambda^{p+q-1} r^{-p-q}\right)
$$

where $c_{ \pm}$is a nonzero complex constant.

Proof. We first prove (i). Recall that

$$
\mathcal{D}_{1}^{p} \mathcal{D}_{2}^{q}(\cos \lambda s)= \begin{cases}\mathrm{O}\left(\lambda^{2 p+2 q}\right) & \text { if } \lambda s \leq 6 \\ \mathrm{O}\left(\lambda^{p+q} s^{-p-q}\right) & \text { if } \lambda s \geq 6\end{cases}
$$

hence $\mathcal{D}_{1}^{p} \mathcal{D}_{2}^{q}(\cos \lambda s)=\mathrm{O}\left(\lambda^{2 p+2 q-1-\varepsilon} s^{-1-\varepsilon}\right)$ in both cases. By combining this estimate with

$$
\sinh s \asymp s, \quad \text { and } \quad \cosh s-\cosh r \asymp s^{2}-r^{2},
$$

and by performing an elementary change of variables, we reach our conclusion:

$$
|\theta(\lambda, r)| \lesssim \lambda^{2 p+2 q-1-\varepsilon} \int_{r}^{6} d s s^{-\varepsilon}\left(s^{2}-r^{2}\right)^{-\frac{1}{2}} \leq \lambda^{2 p+2 q-1-\varepsilon} r^{-\varepsilon} \underbrace{\int_{1}^{+\infty} d s s^{-\varepsilon}\left(s^{2}-1\right)^{-\frac{1}{2}}}_{<+\infty} .
$$

We next prove (ii). Recall that

$$
\mathcal{D}_{1}^{p} \mathcal{D}_{2}^{q}\left(e^{ \pm i \lambda s}\right)=\frac{( \pm i \lambda)^{p+q}}{(\sinh s)^{p}(\sinh s / 2)^{q}} e^{ \pm i \lambda s}+\mathrm{O}\left(\lambda^{p+q-1} s^{-p-q-1}\right)
$$

The remainder's contribution to $\theta^{ \pm}(\lambda, r)$ is estimated as above:

$$
\int_{r}^{6} d s \frac{\sinh s}{\sqrt{\cosh s-\cosh r}} \lambda^{p+q-1} s^{-p-q-1} \lesssim \lambda^{p+q-1} \int_{r}^{6} d s s^{-p-q}\left(s^{2}-r^{2}\right)^{-\frac{1}{2}} \lesssim \lambda^{p+q-1} r^{-p-q} .
$$

In order to handle the contribution of $\frac{( \pm i \lambda)^{p+q}}{(\sinh s)^{p}(\sinh s / 2)^{q}} e^{ \pm i \lambda s}$ we observe that, since $r \leq s \leq$ 6, this term is comparable to $\frac{( \pm i \lambda)^{p+q}}{(\sinh s)^{p+q}} e^{ \pm i \lambda s}$ and we conclude as in [4, Lemma C.1].

From now on, the discussion of Subcase 2.b is similar to Subcase 2.a. On one hand, by applying Lemma C.1.i with $p=\frac{k+1}{2}$ and $q=\frac{m}{2}$, we obtain

$$
\int_{1}^{\frac{6}{r}} d \lambda \chi_{\infty}(\lambda) \lambda^{-\tau}\left(\lambda^{2}+\frac{\widetilde{Q}^{2}}{4}\right)^{\frac{\tau-\sigma}{2}} e^{i t \lambda} \theta(\lambda, r)=\mathrm{O}\left(r^{k+m-\operatorname{Re} \sigma+1}\right)=\mathrm{O}\left(r^{-\frac{n-1}{2}}\right) .
$$

On the other hand, by expanding

$$
\lambda^{-\tau}\left(\lambda^{2}+\frac{\widetilde{Q}^{2}}{4}\right)^{\frac{\tau-\sigma}{2}}=\lambda^{-\sigma}\left(1+\frac{\widetilde{Q}^{2}}{4 \lambda^{2}}\right)^{\frac{\tau-\sigma}{2}}=\lambda^{-\frac{n+1}{2}-i \operatorname{Im} \sigma}+\mathrm{O}\left(|\sigma| \lambda^{-\frac{n+1}{2}-2}\right) \quad \forall \lambda \geq 2
$$


and $\theta^{ \pm}(\lambda, r)$ according to Lemma C.1.ii, we have

$$
\begin{aligned}
& \frac{e^{\sigma^{2}}}{\Gamma(-i \operatorname{Im} \sigma)} \int_{\frac{6}{r}}^{+\infty} d \lambda \chi_{\infty}(\lambda) \lambda^{-\tau}\left(\lambda^{2}+\frac{\widetilde{Q}^{2}}{4}\right)^{\frac{\tau-\sigma}{2}} e^{i t \lambda} \theta^{ \pm}(\lambda, r) \\
& =c_{ \pm}\left(I_{ \pm}+I I_{ \pm}\right)(\sinh r)^{\frac{1-n}{2}}+\mathrm{O}\left(r^{\frac{5}{2}-\frac{n}{2}}\right)
\end{aligned}
$$

where $I_{ \pm}$and $I I_{ \pm}$denote the integrals (61) and (62), which are uniformly bounded and whose sum is equal to

$$
\frac{e^{\sigma^{2}}}{\Gamma(-i \operatorname{Im} \sigma)} \int_{\frac{6}{r}}^{+\infty} d \lambda \lambda^{-1-i \operatorname{Im} \sigma} e^{i(t \pm r) \lambda} .
$$

As a conclusion, we obtain again

$$
\left|\widetilde{w}_{t}^{\infty}(r)\right| \lesssim r^{-\frac{n-1}{2}} \lesssim t^{-\frac{n-1}{2}}
$$

Remark C.3. In order to estimate the wave kernel for small time, we might have used the Hadamard parametrix [19, § 17.4] instead of spherical analysis.

\section{REFERENCES}

[1] J.-Ph. Anker, E. Damek, C. Yacoub, Spherical analysis on harmonic AN groups, Ann. Sc. Norm. Super. Pisa 33 (1996), 643-679

[2] J.-Ph. Anker, V. Pierfelice, Nonlinear Schrödinger equation on real hyperbolic spaces, Ann. Inst. H. Poincaré (C) Non Linear Analysis 26 (2009), 1853-1869

[3] J.-Ph. Anker, V. Pierfelice, M. Vallarino, Schrödinger equations on Damek-Ricci spaces, to appear in Comm. Part. Diff. Eq.

[4] J.-Ph. Anker, V. Pierfelice, M. Vallarino, Nonlinear wave equation on real hyperbolic spaces, preprint [hal-00525251, arXiv:1010.2372]

[5] F. Astengo, A class of $L^{p}$-convolutors on harmonic extensions of $H$-type groups, J. Lie Theory (5) 9 (1995), 147-164

[6] M.G. Cowling, A.H. Dooley, A. Korányi, F. Ricci, H-type groups and Iwasawa dwcompositions, Adv. Math. 87 (1991), 1-41

[7] M.G. Cowling, A.H. Dooley, A. Korányi, F. Ricci, An approach to symmetric spaces of rank one via groups of Heisenberg type, J. Geom. Anal. 8 (1998), 199-237

[8] E. Damek, F. Ricci, A class of nonsymmetric harmonic Riemannian spaces, Bull. Amer. Math. Soc. 27 (1992), 139-142

[9] E. Damek, F. Ricci, Harmonic analysis on solvable extensions of H-type groups, J. Geom. Anal. 2 (1992), 213-248

[10] P. D'Ancona, V. Georgiev, H. Kubo, Weighted decay estimates for the wave equation, J. Diff. Eq. 177 (2001), 146-208

[11] P. D'Ancona, L. Fanelli, Decay estimates for the wave and Dirac equations with a magnetic potential, Comm. Pure Appl. Math. 60 (2007), 357-392

[12] P. D'Ancona, L. Fanelli, Strichartz and smoothing estimates of dispersive equations with magnetic potentials, Comm. Partial Diff. Eq. 33 (2008), 1082-1112

[13] I.M. Guelfand, G.E. Chilov, Les distributions, tome I, Dunod (1962)

[14] V. Georgiev, Semilinear hyperbolic equations, Mem. Math. Soc. Japan 7 (2000)

[15] V. Georgiev, H. Lindblad, C. Sogge, Weighted Strichartz estimates and global existence for semilinear wave equations, Amer. J. Math. 119 (1997), 1291-1319 
[16] J. Ginibre, G. Velo, Generalized Strichartz inequalities for the wave equation, J. Funct. Anal. 133 (1995), 50-68

[17] C.S. Herz, Sur le phénomène de Kunze-Stein, C. R. Acad. Sci. Paris Sér. A 271 (1970 ), 491-493

[18] L.V. Hörmander, The analysis of linear partial differential operators I (distribution theory and Fourier analysis), Springer-Verlag (1983, 1990, 2003)

[19] L.V. Hörmander, The analysis of linear partial differential operators III (pseudo-differential operators), Springer-Verlag (1985, 1994, 2007)

[20] A.D. Ionescu, Fourier integral operators on noncompact symmetric spaces of real rank one, J. Funct. Anal. 174 (2000), 274-300

[21] F. John, Blow-up of solutions of nonlinear wave equations in three space dimensions Manuscripta Math. 28 (1979), 235-265

[22] A. Kaplan, Fundamental solutions for a class of hypoelliptic PDE generated by composition of quadratic forms, Trans. Amer. Math. Soc. 258 (1975), 145-159

[23] M. Keel, T. Tao, Endpoint Strichartz estimates, Amer. J. Math. 120 (1998), 955-980

[24] S. Klainerman, G. Ponce, Global, small amplitude solutions to nonlinear evolution equations, Comm. Pure Appl. Math. 36 (1983), 133-141

[25] T.H. Koornwinder, Jacobi functions and analysis on noncompact semisimple Lie groups, in Special functions (group theoretical aspects and applications), R.A. Askey \& al. (eds.), Reidel (1984), $1-85$

[26] H. Lindblad, C. Sogge, On existence and scattering with minimal regularity for semilinear wave equations, J. Funct. Anal. 130 (1995), 357-426

[27] V. Pierfelice, Weighted Strichartz estimates for the Schrödinger and wave equations on DamekRicci spaces, Math. Z. 260 (2008), 377-392

[28] F. Ricci, The spherical transform on harmonic extensions of $H$-type groups, Rend. Sem. Mat. Univ. Polit. Torino 50 (1992), 381-392

[29] T. Sideris, Nonexistence of global solutions to semilinear wave equations in high dimensions, J. Differential Equations 52 (3) (1984), 378-406

[30] W. Strauss, Nonlinear Wave Equations, CBMS Reg. Conf. Ser. Math. 73, Amer. Math. Soc. (1989)

[31] D. Tataru, Strichartz estimates in the hyperbolic space and global existence for the semilinear wave equation, Trans. Amer. Math. Soc. 353 (2001), 795-807

[32] H. Triebel, Theory of function spaces II, Monographs Math. 84, Birkhäuser (1992)

Université d’Orléans \& CNRS, Fédération Denis Poisson (FR 2964) \& Laboratoire MAPMO (UMR 6628), BÂtiment de mathématiques - Route de Chartres, B.P. 6759 45067 OrlÉANS CEDEX 2 - France

E-mail address: anker@univ-orleans.fr

Université d'Orléans \& CNRS, FÉdération Denis Poisson (FR 2964) \& Laboratoire MAPMO (UMR 6628), BÂtiment de mathématiques - Route de Chartres, B.P. 6759 45067 ORLÉANS CEDEX 2 - FRANCE

E-mail address: vittoria.pierfelice@univ-orleans.fr

Universita' di Milano - Bicocca, Dipartimento di Matematica e Applicazioni, Via Cozzi 53 - 20125 Milano - ItAliA

E-mail address: maria.vallarino@unimib.it 